\title{
Conference Program and Abstracts of the 9th Annual Conference of the Special Interest Group in Neuropsychological Rehabilitation of the World Federation for NeuroRehabilitation (WFNR) \\ 2-3 July, 2012 \\ Bergen, Norway
}

\section{OPENING ADDRESS}

\section{Tuberculous Meningitis: What Neuropsychologists Should Know}

Wilson, Barbara A. ${ }^{1}$

${ }^{1}$ The Oliver Zangwill Centre, Princess of Wales Hospital, Ely, UK

$\boldsymbol{R}$ ackground and Aims: Tuberculous meningitis (TBM) is a bacterial in$\mathcal{B}$ fection of the meninges and the most common form of central nervous system tuberculosis. The infection usually begins elsewhere in the body, typically the lungs, and then travels through the bloodstream to the meninges where small abscesses are formed. When these abscesses burst TBM occurs. First described by Whytt (1768), it is common in poorer countries but unusual in the developed world. This presentation aims to alert neuropsychologists and other health service professionals to the neuropsychological manifestations of the disease and to the typical picture of recovery. Methods: A literature search was carried out to determine the presenting symptoms, treatment and cognitive sequelae of TBM. Three patients, recently assessed in the United Kingdom, are presented to illustrate the cognitive profile and the recovery process. Results: Recovery is often delayed and may be seen after a lengthy period of reduced consciousness, for example more than two years post diagnosis. The recovery might be associated with cessation of pharmacological treatment. Initially patients need to be assessed with tests for people in states of reduced consciousness, although as recovery continues, more traditional tests can be administered. A lengthy period of retrograde amnesia is commonly seen. Discussion and Conclusions: Although uncommon in the developed world, neuropsychologists may occasionally be asked to assess and treat patients with TBM. It is important, therefore, that they are aware of the typical picture, what assessments to use and what to look out for during the recovery process.

Correspondence: Barbara Wilson; barbara.wilson00@gmail.com 


\title{
SESSION 1: SYMPOSIUM POSTTRAUMATIC AMNESIA (PTA): RETRACING OUR STEPS TO FIX PROBLEMS AND AVOID PITFALLS
}

\section{Problems with the theoretical construct: PTA vs. delirium}

\author{
Kean, Jacob ${ }^{1,3}$; Abell, Malene ${ }^{2}$ and Parrott, Devan ${ }^{3}$ \\ ${ }^{1}$ Department of Physical Medicine and Rehabilitation, Indiana University School of Medicine, USA \\ ${ }^{2}$ Department of Psychological and Brain Sciences, Indiana University, USA \\ ${ }^{3}$ Department of Research, Rehabilitation Hospital of Indiana, USA
}

\begin{abstract}
$B$ ackground: Posttraumatic amnesia (PTA) was a term coined by Symonds and Russell to operationalize the end of the acute impairment of consciousness that follows traumatic brain injury (TBI). Over time, the field has adopted the operational endpoint as the construct, ignoring the breadth of symptoms during this period of recovery, which are more characteristic of delirium than amnesia. PTA and delirium differ not only in conceptual breadth but also in their treatment of fluctuation, which is a DSM-IV diagnostic criterion for delirium but ignored as a conceptual element in PTA. Aim: We sought to investigate: 1) the validity of a broader set of indicators that were consistent with the construct of delirium (e.g., attention, executive-semantic ability), and 2) the incidence and relevance of assessment-to-assessment fluctuation, defined as a change in direction in Orientation $\log (\mathrm{O}-\mathrm{Log})$ total score. Method: A retrospective cohort study of 90 patients admitted for inpatient rehabilitation following TBI was conducted. Initial Rasch model item calibrations were conducted with $257 \mathrm{O}-\mathrm{Log}$ scores from this sample and then recalibrated using a broader set of indicators. The occurrence and magnitude of fluctuations were studied in a subset of 76 patients with three or more assessments. Results: Rasch model calibrations showed the broader set of indicators to be valid and the latent construct more fully represented by these items, as indicated by sufficient item fit statistics and improved measure reliability. The incidence of fluctuation in orientation during the inpatient rehabilitation hospitalization was $76 \%$ (58/76 inpatients). Comparison of groups with fluctuating vs. stable O-Log scores showed the fluctuating group had significantly lower FIM total scores $(t=-2.23, p=.029)$. Finally, the addition of the magnitude of fluctuation in a multiple regression model significantly improved prediction of discharge FIM scores over a model that included age, initial Glasgow Coma Scale score, and PTA duration $\left(\mathrm{F}=7.11, \mathrm{p}=.000\right.$, adjusted $\mathrm{R}^{2}=0.34$ vs. $\mathrm{F}=5.74, \mathrm{p}=.002$, adjusted $\left.\mathrm{R}^{2}=.023\right)$. Conclusions: Two key differences between PTA and delirium conceptualisations of early recovery from TBI are the relative breadth of delirium vs. PTA and the importance of fluctuation. Both the review of literature and our analyses suggest conceptualising the period of early recovery following TBI as delirium instead of PTA improves the monitoring of recovery and yields better prognostic information.
\end{abstract}

Correspondence: Jacob Kean; jakean@indiana.edu

\section{Conceptual models of early recovery following brain injury}

\author{
Abell, Malene $^{1}$ and Kean, Jacob ${ }^{2,3}$ \\ ${ }^{1}$ Department of Psychological and Brain Sciences, Indiana University, USA \\ ${ }^{2}$ Department of Physical Medicine and Rehabilitation, Indiana University School of Medicine, USA \\ ${ }^{3}$ Department of Research, Rehabilitation Hospital of Indiana, USA
}

$\boldsymbol{B}$ ackground: Aligning a multidisciplinary care team around a conceptual model is a key factor in successful implementation of critical care 
rehabilitation services. However, critical care and rehabilitation settings currently have different conceptual models of early recovery following brain injury. Aim: The aim of this study was to investigate different descriptive models of the recovery of consciousness that supported either critical care or rehabilitation conceptualisations of early recovery from traumatic brain injury. This involved partially replicating and extending a study by Stuss et al. (1999) investigating memory and attention impairments. Adding measures of executive function, sleep-wake cycle and semantic comprehension and examining the variability in the clearing pattern found by Stuss and colleagues extended the original study. Methods: This was a retrospective analysis of a clinical cohort $(\mathrm{N}=90)$ following traumatic brain injury. A Sign Test with $\mathrm{p}<0.05$ was used to compare time from injury to normal range performance between pairs of measures in group and individual patient data to examine the linearity of recovery using time-to-event analyses. Longitudinal data was used to examine to what extent individual patterns differ from the linear model. Results: At some level, each of the descriptive models demonstrated utility. At a clinically meaningful level, recovery was generally linear but marked by variability and indeterminacy associated with the delirium and critical care conceptualisation of recovery of consciousness. Conclusions: Based on the variability and indeterminacy found in these analyses, delirium appears to be more useful and accurate than posttraumatic amnesia as a conceptual framework for the rehabilitation of persons with TBI in the critical care setting.

Correspondence: Malene Abell; mallholde@indiana.edu

\section{Delirium Phenomenology In TBI Rehabilitation and Palliative Care Cohorts Using Factor Analysis of the DRS-R98}

Abell, Malene ${ }^{1}$ and Kean, Jacob ${ }^{2,3}$

${ }^{1}$ Department of Psychological and Brain Sciences, Indiana University, USA

${ }^{2}$ Department of Physical Medicine and Rehabilitation, Indiana University School of Medicine, USA

${ }^{3}$ Department of Research, Rehabilitation Hospital of Indiana, USA

ackground: Although early recovery after TBI may fit the conceptual
model of delirium it might differ in symptom presentations from delirium
stemming from other aetiologies. Aim: Examine underlying factor structure of
delirium symptoms in a population with TBI compared to other aetiologies us-
ing a comprehensive, well-validated assessment tool. Method: Principal Axis
Factor analysis of the Delirium Rating Scale - Revised-98 (DRS-R98) was em-
ployed to detect latent factors of delirium symptoms. Results: $60.2 \%$ scored
14 on the DRS-R98 severity scale (delirium); $17.7 \%$ were diagnosed with
comorbid dementia. Three factors explained 51.8\% of the total variance in
the total sample with Factor 1 comprising cognitive items, language, thought
process, motor agitation and sleep/wake cycle, Factor 2 comprising affec-
tive lability, delusions, motor agitation and sleep/wake cycle, and Factor 3
comprising motor retardation. Perceptual disturbances/hallucinations did not
load on any factor. Similar results were found when excluding subjects with
TBI or comorbid dementia. TBI patients alone produced a two-factor solution
with cognitive, motor agitation/retardation and sleep/wake cycle loading on
Factor 1 and affective lability, delusions, thought process abnormalities and
sleep/wake cycle loading on Factor 2 . A separate analysis of patients with
comorbid dementia yielded a three-factor solution with cognitive items on
Factor 1 , delusions and affective lability on Factor 2 , and long-term memory, 
sleep/wake cycle and perceptual disturbances/hallucinations on Factor 3. Motor agitation or retardation did not load on any factor in the dementia sample. Conclusion: Delirium symptoms appear to be organised in fairly stable latent factors across aetiologies except for motor items.

Correspondence: Malene Abell; mallholde@indiana.edu

\title{
SESSION 2: ADVANCES IN SINGLE-CASE METHODOLOGY
}

\section{Developing reporting guidelines for single-case experimental designs: the SCRIBE project}

Tate, Robyn ${ }^{1}$; Togher, Leanne²; Perdices, Michael ${ }^{3} ; \mathrm{McDonald} \mathrm{Skye}^{4}$ and Rosenkoetter, Ulrike ${ }^{1}$ on behalf of the SCRIBE Steering Committee

${ }^{1}$ Rehabilitation Studies Unit, Sydney Medical School, University of Sydney, Sydney, Australia

${ }^{2}$ Discipline of Speech Pathology, Faculty of Health Sciences, University of Sydney, Sydney, Australia

${ }^{3}$ Department of Neurology, Royal North Shore Hospital, Sydney, Australia

${ }^{4}$ School of Psychology, University of New South Wales, Sydney, Australia

\begin{abstract}
$B$ ackground and aims: Standards of reporting the results of research in medical journals have been criticised. As a consequence, guidelines have been developed with the aim of providing an agreed-upon set of criteria to improve reporting. An early guideline was the Consolidated Standards of Reporting Trials (CONSORT) Statement for randomized controlled trials. The present report describes the Single-Case Reporting guideline In BEhavioural interventions (SCRIBE). Method: The SCRIBE evolved directly from a related guideline under development (CONSORT Extension for N-of-1 Trials; CENT). Whereas CENT is intended for multiple cross-over, A-B-A-B designs used in medicine, SCRIBE aims to cover the broader range of single-case experimental designs (SCED) used in the behavioural sciences. To develop the SCRIBE, a set of items was derived from the CENT Statement and a method quality SCED Scale, which were subject to two rounds of a web-based Delphi exercise. Results were discussed at a 2-day consensus conference to develop items for the SCRIBE. Results: Each of the SCRIBE Steering Committee, Delphi survey and consensus conference had international representation, and included clinicians, educationalists, methodologists, statisticians, medical librarians and journal editors. Results of the 44-item Delphi surveys were discussed at the consensus conference and a final set of 26 items for the SCRIBE was unanimously agreed by conference participants. Conclusions: The SCRIBE provides a brief and practical 'user's guide' to publishing single-case studies and we anticipate that, as has been demonstrated for the CONSORT Statement, a by-product will be the improved planning and conduct of single-case research.
\end{abstract}

Correspondence: Robyn Tate; rtate@med.usyd.edu.au

\section{The utility of regression analysis in single-case experimental designs}

\author{
Manolov, Rumen ${ }^{1,2}$ and Solanas, Antonio ${ }^{1,2}$ \\ ${ }^{7}$ Department of Behavioural Sciences Methods, Faculty of Psychology, University of Barcelona, Barcelona, \\ Spain \\ ${ }^{2}$ Institute for Research in Brain, Cognition, and Behaviour (IR3C), Barcelona, Spain
}

$B$ ackground and aims: The main regression-based data analytical proposals are reviewed. Special attention is paid to the ease of calculation and interpretation, as well as to statistical soundness. The focus is a recent proposal based on generalised least squares for quantifying effect size (GLS; Maggin 
et al., 2011). We aim to explore the performance of the GLS and compare it to a simple procedure giving practically the same information. Method: The GLS is tested via Monte Carlo sampling in a wide variety of conditions. Two types of effect are simulated (slope and level change), with two different magnitudes. Additionally, the effect of confounding variables is studied: linear and quadratic trend, heterogeneity of variance across phases, and several serial dependence patterns (i.e., constant, phase-specific degree of autocorrelation and phase-specific underlying data generation process). Results: Despite GLS's authors' recommendations for a series of at least 20 data points and the assumption of constant serial dependence across phases, the procedure proves to perform adequately for shorter series and for phase-specific serial dependence, as well as when linear trend is present. The only distortion is due to quadratic trend. Equivalent results are obtained for the simple procedure proposed, which is actually more sensitive to treatment effects. Conclusions: The positive message is that psychologists have several potentially useful data analytical techniques. In order to quantify the difference between a baseline and a treatment phase, we recommend using the simpler procedure, which requires fewer steps and is more straightforward in terms of interpretation.

Correspondence: Rumen Manolov; rrumenov13@ub.edu

\section{SESSION 3: DATABLITZ - SERVICE AND OUTCOME EVALUATION}

\section{Effects of a holistic neuropsychological rehabilitation programme (Intensive Neu- roRehabilitation, INR) on emotional well-being, coping, and quality of life after acquired brain injury}

Holleman, Meike ${ }^{1,2}$; Vink, Martie ${ }^{1}$; Horst, Rick¹; Luijpen, Marijn'; Rienstra, Anne ${ }^{1,3}$ and Schmand, Ben ${ }^{2,3}$

${ }^{1}$ Reade, centre for rehabilitation and rheumatology, Amsterdam, The Netherlands

${ }^{2}$ Department of Neurology, Academic Medical Centre at the University of Amsterdam, Amsterdam, The Netherlands

${ }^{3}$ Program group Brain \& Cognition, Faculty of Social and Behavioural Sciences, University of Amsterdam, Amsterdam, The Netherlands

\footnotetext{
$B$ ackground and aims: The Intensive NeuroRehabilitation (INR) programme is a comprehensive or holistic outpatient neuropsychological rehabilitation service that aims to treat cognitive, emotional, and behavioural changes that persist after acquired brain injury. It consists of four days a week of therapy, during two blocks of seven weeks, with a two-week 'home practice break' in between. Method: Thirty-eight consecutive patients (26 male, 12 female) with acquired brain injury (e.g., traumatic brain injury, stroke, extirpated tumour), referred to the INR programme, were included in a randomised, waiting-list controlled study. Patients were included in the control or experimental group based on time of referral. The waiting-list group underwent two assessments 14 weeks apart before treatment, while the experimental group underwent a pre- and post-treatment assessment. Differences between the preand post-treatment assessment (experimental group) were compared to differences between baseline and pre-treatment assessment (control group). Assessment consisted of neuropsychological tests (attention, memory, executive functioning) and questionnaires regarding psychopathology, coping, quality of life, and awareness of deficit. Results: Significant changes were found in the experimental, but not in the control group, regarding emotional well-being, coping, and quality of life. No changes were evident for the neuropsychological test results. The awareness measures failed to demonstrate clear results.
} 
However, time since injury was longer in the experimental group. Conclusions: Patients with traumatic brain injury were found to benefit significantly from the INR programme with regard to emotional well-being, coping, and quality of life. Time since injury was longer in the experimental group and may be a confound.

Correspondence: Meike Holleman; m.holleman@ reade.nl

\title{
Bringing evidence-based practice into the clinic using the Model for Assessing Treatment Effect (MATE)
}

\author{
Tate, Robyn ${ }^{1}$; Taylor, Christine ${ }^{2}$ and Aird, Vanessa ${ }^{2}$ \\ ${ }^{1}$ Rehabilitation Studies Unit, Sydney Medical School, University of Sydney, Sydney, Australia \\ ${ }^{2}$ Cortex Communication Partners, Sydney, Australia
}

\begin{abstract}
$B$ ackground and aims: Clinical practice is typically characterised by pretreatment assessment, development of therapy goals, and implementation of therapy which may (or may not) continue until therapy goals are achieved. Our observations, in the context of post-acute and community rehabilitation services for traumatic brain injury, are that therapy is generally delivered in the absence of control of extraneous variables and formal evaluation of treatment effect is rarely conducted using behaviour specific measures. Rather, therapeutic effect is often evaluated via goal attainment. These factors make it impossible to determine whether improvement is due to specific treatment effects or other extraneous factors. Method: We developed a model to assess treatment effect (MATE), which is applicable to any rehabilitation discipline and any stage of the rehabilitation process. The MATE incorporates assessment and treatment principles guided by the International Classification of Functioning, Disability and Health and single-case methodology. Results: The MATE is hierarchical and comprises seven levels, ranging from no therapy (Level 0) to therapy using single-case experimental methodology, in which an intervention is delivered in a planned and systematic manner to a patient who serves as his/her own control (Level 6). The intervening levels (1-5) have varying configurations of pre- and post-therapy evaluation using generic and specific measures, but treatment is uncontrolled. Conclusions: We have applied the MATE in auditing a clinical service and it differentiates well among various levels of sophistication of service delivery. We propose that use of the MATE within the clinical setting has potential to benchmark quality of clinical services.
\end{abstract}

Correspondence: Robyn Tate; rtate@med.usyd.edu.au

\section{The health economy of holistic rehabilitation: four case studies}

\author{
Winegardner, Jill ${ }^{1}$ and Ashworth, Fiona ${ }^{1}$ \\ ${ }^{1}$ Oliver Zangwill Centre, Ely, UK
}

$\boldsymbol{R}$ ackground and aims: Neuropsychological rehabilitation programmes have come under increasing financial pressure to demonstrate not only improvement in quality of life but also cost savings to society (Worthington et al, 2006). We aim to demonstrate that holistic rehabilitation for complex consequences of brain injury is effective as well as cost-effective. Method: We evaluate the "return on investment" in four cases, focusing on projected savings in the areas of mental health care, health services, social services, forensic care, and the tax base. The four cases involve clients seen within 
an inter-disciplinary holistic rehabilitation programme. They reflect a crosssection of typical clients in terms of age, gender, diagnosis, and complexity of needs. Results: Case 1 describes a young woman whose attempted suicide cost $£ 50,000$ in mental health and medical costs. Case 2 describes a young man on the verge of further imprisonment due to poor anger management who avoided incarceration with the support of strategies learned in rehabilitation. Case 3 reports the savings in social services and support worker costs for a woman who went from physical dependence on others to full independence following rehabilitation. Case 4 describes a lead engineer unable to work after a severe concussion who returned to work after rehabilitation and subsequently contributed substantially to the tax base. Discussion and Conclusions: Investment in holistic rehabilitation has potential for huge savings in government costs in a young brain injured population. Clinical programmes should look at health economy measures as well as quality of life measures in the current fiscal environment.

Correspondence: Jill Winegardner; Jill.winegardner@ozc.nhs.uk

\section{The relationship between TBI history and treatment outcome in addictions treat- ment: Building the case for integrated care}

Lemsky, Carolyn ${ }^{1}$; Gargaro, Judy'; Godden, Tim²; James, Dennis ${ }^{2}$ and Ann Hilliard ${ }^{2}$

${ }^{1}$ Community Head Injury Resource Services of Toronto, Toronto Ontario (CHIRS)

${ }^{2}$ Centre for Addictions and Mental Health, Toronto Ontario (CAMH)

$\boldsymbol{R}$ ackground and aims: The aim of the current study was to determine the 3 extent to which acquired brain injury (ABI) affects treatment outcomes in usual addictions treatment at a large tertiary addictions and mental health facility. Data will be used to inform policy and funding allocation. Method: Screening using the Ohio TBI identification method (Corrigan \& Bogner, 2007) was undertaken at the Centre for Addiction and Mental Health's main urban campus and included intake assessments for general as well as specialized (e.g. concurrent disorders) addictions programming. Outcome measures included the Addiction Severity Index as well as treatment duration (drop-out) and program assignment. Results: In the first month of data collection $(N=87)$, $38 \%(n=33)$ were identified as having at least one brain injury. Of these, $15 \%$ $(n=5)$ had two or more injuries. Twenty-one (24\% of the total sample) reported loss of consciousness. Of those reporting brain injury, $48.5 \%(n=16)$ reported having received a mental health diagnosis in their life time; $12 \%(n=5)$ in the last month. Data from the first four months of screening and outcomes at three and six months will be presented. Conclusions: Preliminary data are consistent with findings from US studies which found similar rates of ABI and identified $\mathrm{ABI}$ as a risk factor for lifetime and new mental health diagnosis. We hypothesize that a subset of individuals with a history of ABI are at risk for poor outcomes in the absence of systematic program adaptation. To our knowledge, this is the first examination of outcome in a mainstream addictions program using $\mathrm{ABI}$ as a controlling factor.

Correspondence: Carolyn Lemsky; clemsky@CHIRS.com 


\title{
Service evaluation of holistic neuropsychological rehabilitation: Impact on dysex- ecutive symptoms and carer strain following traumatic and non-traumatic brain injuries
}

Goodwin, Rachel ${ }^{1,2}$ and Bateman, Andrew ${ }^{1}$

${ }^{1}$ Institute of Work, Health and Organisations, The University of Nottingham, Nottingham, UK

${ }^{2}$ The Oliver Zangwill Centre for Neuropsychological Rehabilitation, Ely, UK

\begin{abstract}
$\boldsymbol{R}$ ackground and aims: Deficits in executive functioning (EF) are common following acquired brain injury (ABI). Patients encounter problems in everyday functioning, and their families experience significant strain. Whilst literature confirms benefits of cognitive rehabilitation for executive dysfunction, this study evaluates the effectiveness of holistic neuropsychological rehabilitation specifically on EF deficits and carer strain. Literature suggests the aetiology of brain injury moderates the effectiveness of specific interventions following ABI. This study assesses whether aetiology interacts with the effects of rehabilitation. Method: Sixty-six chronic outpatients with ABI (50 with traumatic brain injury; 16 with non-traumatic brain injuries) attended 24-weeks of holistic neuropsychological rehabilitation. A repeatedmeasures design determined the effect of rehabilitation on EF and carer strain, as part of service evaluation. Outcome measures comprised the dysexecutive questionnaire (DEX/DEX-I) and carer strain index (CSI), applied pre- and post-rehabilitation. Results: Rehabilitation benefited clients and carers in 5/6 DEX/DEX-I, and 2/3 CSI subscales, $(\mathrm{p}<.05)$. Results showed significant improvement in items sampling clients' planning and insight, and carers' personal upset and lifestyle adjustments. However, items covering clients' aggression and carers' financial strain did not improve. Secondary analysis showed an effect of aetiology on rehabilitation, demonstrated on DEX-I metacognitive scale; clients with traumatic injuries significantly improved while clients with non-traumatic injuries showed no benefit. Conclusions: Neuropsychological rehabilitation is effective in reducing client and carer reports of dysexecutive behaviours and carer strain. Rehabilitation can benefit ABI clients and their families, even after the spontaneous recovery period. Also implicated is the importance of considering aetiology in practice as results on the DEX-I metacognitive scale were masked by aetiological group differences.
\end{abstract}

Correspondence: Rachel Goodwin; rachelanngoodwin@gmail.com

\section{SESSION 4: DATABLITZ - TREATMENT TECHNIQUES AND CONSIDERATIONS}

\section{Can brief Goal Management Training improve performance on simple computerised prospective memory tasks? A randomised study}

\author{
Baylan, Satu ${ }^{1,2}$ and Evans, Jonathan ${ }^{1,2}$ \\ ${ }^{1}$ Academic Unit of Mental Health \& Wellbeing, University of Glasgow, Glasgow, UK \\ ${ }^{2}$ Sackler Institute of Psychobiological Research, University of Glasgow, Glasgow, UK
}

\begin{abstract}
$B$ ackground and aims: Deficits in prospective memory (PM) affect the ability to carry out future intentions, compromising independent functioning. PM complaints are common following neurological illness yet the evidence-base in support of PM interventions is limited. This study investigated whether brief Goal Management Training combined with Implementation Intentions (GMTii) can improve performance on computerised PM tasks developed for use in neuroimaging and shown to correlate with everyday
\end{abstract}


functioning. Method: Thirty neurologically healthy individuals were randomly assigned to GMTii or control training. Participants completed a computerised task pre- and post-training that involved carrying out an ongoing task into which a PM task was embedded. In addition, a novel task of similar nature was completed post-training to assess generalisability of any effect. Results: The overall performance of the two groups did not differ significantly pre-training, $(\mathrm{U}=97, \mathrm{z}=-.640, \mathrm{~ns}, \mathrm{r}=-.12$ ). Post-training, the GMTii group showed significantly better performance on the computerised PM task compared to the control group - the GMTii group showed less performance decay over the duration of the task compared to the control group $(\mathrm{t}=2.61$ [28], $\mathrm{p}<.05)$. On a novel task, the GMTii group performed better on some but not all measures. Conclusions: The results indicate that brief GMTii can reduce the occurrence of PM slips on tasks designed for use with neuroimaging methods and which have been shown to correlate with everyday functioning. This suggests that these measures may be useful outcome measures, affording the opportunity to study the neural impact of GMTii interventions.

Correspondence: Satu Baylan; s.baylan@clinmed.gla.ac.uk

\section{The effect of compensatory scanning training on mobility in hemianopia patients}

De Haan, Gera'; Heutink, Joost ${ }^{1,2}$; Melis-Dankers, Bart ${ }^{2}$; Tucha, Oliver ${ }^{1}$ and Brouwer, Wiebo $^{1}$

${ }^{1}$ Department of Neuropsychology across the Life-Span; University of Groningen; Groningen; The Netherlands ${ }^{2}$ Royal Dutch Visio, Haren, The Netherlands

$\boldsymbol{R}$ ackground and aims: Homonymous hemianopia, the most common form $\boldsymbol{B}$ of Homonymous Visual Field Defects (HVFD), refers to a loss of perception for half the visual field, affecting both eyes, due to acquired postchiasmatic brain injury. Because of a disorganised visual search strategy, patients with HVFD have particular difficulties with visual exploration and mobility. Method: A new Compensatory Scanning Training protocol has been developed, which aims to improve awareness, scanning and mobility in daily life. The effect of this training protocol was examined in 30 hemianopia patients. Among other tests, we administered a simulated driving task which required responding to peripheral stimuli during driving, an ecologically valid obstacle course, and the Independent Mobility Questionnaire, reflecting mobility in daily life. The examinations were done the week before and the week after training and 16 of these patients were examined three months before start of the training as well. Results: After training, the reaction times for stimuli in the blind periphery decreased and less stimuli were omitted on the blind side, as measured with the simulated driving task. Walking through the obstacle course while performing a cognitive dual task took less time after training. Furthermore, patients reported a decrease in daily life mobility problems. The relationship between the three different tests will be discussed, as well as the results for the patients who also participated in the early pre-measurement. Discussion and Conclusions: Compensatory Scanning Training may have beneficial effects on scanning and mobility in hemianopia patients.

Correspondence: Gera De Haan; G.A.de.Haan@rug.nl 


\title{
Investigating the use of iPad and App to deliver Semantic Feature Analysis (SFA) Treatment in clients with aphasia
}

\author{
Kearns, Áine ${ }^{1}$; Franklin, Sue ${ }^{1}$ and Higgins Conor $^{2}$ \\ ${ }^{1}$ Department of Clinical Therapies, University of Limerick, Limerick, Ireland \\ ${ }^{2}$ Interactive Design Centre, University of Limerick, Limerick, Ireland
}

\begin{abstract}
$B$ ackground and aims: Custom made Speech and Language Therapy (SLT) software for use in aphasia therapy has historically been restricted by the lack of a large viable market (Yu-kong Choe et al, 2010). New, user friendly advances in software and hardware mean that flexible and no-cost programmes for use with people with aphasia are now possible. The aim of this study was to examine the potential benefits of delivering a word retrieval treatment, Semantic Feature Analysis (SFA; Boyle 2010), in the form of iPad and App. Method: An item-specific design was used with single case studies $(n=4)$. Treated, related controls and unrelated controls were assessed twice before and once after therapy. Participants received 8 sessions of therapy in a 4 week period and were encouraged to use the iPad App at home at least 5 times per week. Results: Post-test analysis indicated no significant change in ability to spontaneously name words taking first response only. Including self corrected words as correct, Participant 3 showed significant improvement for treated words only $(p=0.0127)$. A significant improvement in Participant 4's naming of treated words $(p=0.0026)$ was accompanied by more modest signs of generalisation to all untreated words $(p=0.0227)$. Participants 1 and 2 did not show significant change following therapy. Conclusions: Results suggest that SFA can be delivered successfully via iPad and App, although it is not clear why only two of the participants improved. The pattern of generalisation demonstrated by Participant 4 supports the notion that SFA works as a selfcueing strategy rather than enriching semantic knowledge (Boyle 2010).
\end{abstract}

Correspondence: Áine Kearns; Aine.Kearns@ul.ie

\section{Cognitive rehabilitation in Parkinson's disease: aspects of feasibility and goal set- ting}

Vlagsma, Thialda $\mathrm{T}^{1}$; van Laar, Teus ${ }^{2}$; Tucha, Oliver ${ }^{1}$; Koerts, Janneke ${ }^{1}$; Schönherr, Marleen $^{3}$; Gerritsen, Marleen ${ }^{4}$ and Spikman, Jacoba ${ }^{1,4}$

${ }^{7}$ Department of Clinical \& Developmental Neuropsychology, University of Groningen, Groningen, The Netherlands

${ }^{2}$ Department of Neurology, University Medical Center Groningen, Groningen, The Netherlands

${ }^{3}$ Department of Neuro-rehabilitation, Rehabilitation center Beatrixoord, Haren, The Netherlands

${ }^{4}$ University Medical Center Groningen, Department of Neuropsychology, Groningen, The Netherlands

$\boldsymbol{B}$ ackground and aims: When cognitive rehabilitation programs are discussed in research papers, the emphasis is generally on their effectiveness, however information about the feasibility of the treatment is also important but often missing (Spikman et al. 2010). A Randomized Controlled Trial (RCT) is underway on the effectiveness and feasibility of treatments for executive dysfunctioning in Parkinson's Disease (PD). Treatments consist of either a Strategic Executive Training (ReSET) or computer training for aspects of attention (Cogniplus). The focus of this paper is on aspects of feasibility. In addition, to gain a better insight into the aspects of executive functioning that patients experience as most restricting, various treatment goals have been differentiated. Method: To determine the feasibility of the two treatment programs in the RCT (Strategic Executive Training and Cogniplus), we analyzed 
the following measures: drop-out rate, mean period of treatment, frequency of sessions per week, fatigue and satisfaction. Furthermore, patients' treatment goals were studied. Results: The number of sessions per week differed slightly between patients due to constraints such as work or mental load capacity, but to date all patients have completed the treatment program, indicating that it is feasible. The level of fatigue over all sessions ranged from average to above average, but patients were highly satisfied. Treatment goals were most frequently related to improving planning of daily activities and increasing household activities. Conclusions: The results of this study can be used to adjust treatment protocols to patients' needs and capabilities. In general, feasibility studies are important to continue development and improvement of cognitive rehabilitation programs.

Correspondence: Thialda Vlagsma; t.t.vlagsma@rug.nl

\title{
First steps in designing an easy-to-use videophone for persons with dementia
}

Boman, Inga-Lill ${ }^{1}$; Rosenberg, Lena ${ }^{1}$; Lundberg, Stefan ${ }^{2}$ and Nygård, Louise ${ }^{1}$

${ }^{1}$ Division of Occupational Therapy, Karolinska Institutet, Stockholm, Sweden

${ }^{2}$ Royal Institute of Technology, School of Technology and Health, Handen, Sweden

\begin{abstract}
$\boldsymbol{B}$ ackground and aims: People with dementia often have difficulties in $B$ managing to use an ordinary telephone. Therefore, people with dementia might need an easy-to-use videophone. Little research has been conducted to develop communication technology for people with dementia. The aim of this study was to design an intuitive user-interface for a videophone for people with dementia. Method: Literature reviews and focus groups were used to identify users' potential for managing a videophone and to gather recommendations regarding the design of the videophone to meet the needs and skills of people with dementia. Existing videophones in Sweden were examined regarding potential to fit users with dementia. Results: This lead to detailed identification of cognitive, physical and psychosocial challenges that people with dementia will probably have when using communication technology, to formulate a requirement specification and develop a design concept. The results gave useful information for further work to develop a prototype to be tested by people with dementia and their significant others. Conclusions: Preliminary data reveal that a videophone for people with dementia has the possibilities to support communication and social interaction, prevent social isolation, help people to feel safe and improve quality of life for people with dementia and their significant others. The requirement specification might also facilitate design of other products for people with dementia, particularly in the field of communication technology.
\end{abstract}

Correspondence: Inga-Lill Boman; inga-lill.boman@ki.se 


\title{
Autobiographical memory facilitation in multiple sclerosis: a neuropsychological and neuroimaging approach
}

Ernst, Alexandra'; Botzung, Anne"; Voltzenlogel, Virginie ${ }^{2}$; Blanc, Frédéric ${ }^{1,3}$; De Seze, Jérôme ${ }^{1,3}$; Sellal, François ${ }^{4}$ and Manning, Lilianne' ${ }^{1}$.

${ }^{1}$ Laboratoire d'Imagerie et de Neurosciences Cognitives, University of Strasbourg, Strasbourg, France

${ }^{2}$ Laboratoire Octogone, University le Mirail, Toulouse, France

${ }^{3}$ Hôpitaux Universitaires de Strasbourg, Strasbourg, France

${ }^{4}$ Hôpitaux Universitaires de Colmar, Colmar, France

\begin{abstract}
$B$ ackground and aims: In a clinical-first study, we showed an autobiographical memory (AbM) impairment in non-depressed multiple sclerosis (MS) patients. Data suggested an access deficit. On this basis, a mental visual imagery (MVI)-based facilitation programme was created. Aim: To assess the efficacy of this programme for supporting AbM retrieval, by testing both clinical and cerebral network changes before and after facilitation. Method: A first group of MS patients underwent neuropsychological assessment and a stringent $\mathrm{AbM}$ test, followed by our facilitation programme, which consisted of MVI exercises of increasing difficulty. A post-facilitation reassessment of $\mathrm{AbM}$ was conducted afterwards. Ten controls also received the AbM test twice. A second group of patients is currently following the same procedure within a pre-/post-facilitation fMRI study design. fMRI tasks are then conducted before and after the facilitation programme and involve evoking past personal events. Results: To date, all the patients who followed the facilitation programme have shown a significant improvement on the AbM test, while the controls' scores remained stable. Furthermore, patients' feedback supported the beneficial effects of the intervention. fMRI data on the first two patients showed enhanced activity in posterior cerebral regions after the facilitation programme. Conclusions: Clinically, this programme based on MVI has been found to be beneficial to access AbMs in these patients. Our fMRI study could confirm these findings, at least in some patients.
\end{abstract}

Correspondence: Alexandra Ernst; alexandra.ernst@etu.unistra.fr

\section{SESSION 5: INTERVENTION OUTCOMES AND THE EVIDENCE BASE}

\section{Efficacy of a Multi-Family Group Intervention following brain injury}

Ponsford, Jennie ${ }^{1,2,3}$; Couchman, Grace ${ }^{1}$; Kelly, Amber ${ }^{1}$ and McMahon, Genevieve ${ }^{1}$

${ }^{7}$ School of Psychology and Psychiatry, Monash University, Melbourne, Australia

${ }^{2}$ Monash-Epworth Rehabilitation Research Centre, Epworth Hospital, Melbourne, Australia

${ }^{3}$ National Trauma Research Institute, Alfred Hospital, Melbourne, Australia

R ackground and Aims: Multi-family group interventions (MFG) (McFar$B$ lane 2003) involve participation of individuals with a health condition and their family members in facilitated groups incorporating psycho-education, problem-solving and social networking. The Headstart program examined the impact of a modified 12-week MFG program for families affected by acquired brain injury $(\mathrm{ABI})$ on family functioning, community integration and emotional distress. Method: The study employed a waitlist control design, with a 12-week baseline, followed by 12-week group participation. Participants included 41 individuals with brain injury ( $83 \% \mathrm{TBI}$ ), with a mean age of 39.36 years $(\mathrm{SD}=14.53)$ and 41 family members with a mean age of 53.31 years $(\mathrm{SD}=12.02)$, predominantly parents $(53.7 \%)$ or spouses/partners $(31.7 \%)$. Outcome measures completed at the beginning and end of each phase by both the person with a brain injury and their family member included the 
Family Assessment Device (FAD), Community Integration Questionnaire (CIQ), Community Integration Measure (CIM) and Hospital Anxiety and Depression Scale (HADS). Data analyses included repeated measures ANOVA with post hoc comparisons. Results: Relatives demonstrated a significant reduction in unhealthy family functioning on the FAD following the MFG relative to baseline. They also showed an increase in both social and overall community integration. There were no significant changes in anxiety and depression on the HADS. No statistically significant changes were evident for individuals with ABI. Discussion: A systemic approach to post ABI adjustment may result in improved family functioning and community integration for family members, with less benefit evident on measures used for the injured participants. Benefits may have been limited by the relatively short duration of intervention.

Correspondence: Jennie Ponsford; jennie.ponsford@monash.edu

\title{
The Scottish Intercollegiate Guideline Network (SIGN) guideline on rehabilitation of cognitive and mood disorders after brain injury
}

\author{
Evans, Jonathan ${ }^{1}$, Jack, Roisin ${ }^{2}$ and Harrison, Andrew ${ }^{3}$ \\ ${ }^{1}$ Institute of Health \& Wellbeing, University of Glasgow, Glasgow, UK \\ ${ }^{2}$ Craig Court Rehabilitation Unit, Aberdeen, UK \\ ${ }^{3}$ Astley Ainslie Hospital, Edinburgh, UK
}

$\boldsymbol{R}$ ackground and aims: The Scottish Intercollegiate Guideline Network develops evidence based clinical practice guidelines for the National Health Service (NHS) in Scotland. We will describe and discuss the process of guideline development in the area of brain injury rehabilitation, focusing on rehabilitation of cognitive and mood disorders. Method: A systematic review process was undertaken. Following systematic searching, papers relevant to the review topics were selected and rated for methodological quality by three reviewers. Taking into account methodological quality, considered judgements and specific recommendations were developed in each of the review areas. Draft recommendations were subjected to peer review. Results: In relation to cognitive rehabilitation, several good quality systematic reviews exist and recommendations were identified in each of the following domains: memory, attention, executive functioning, and visuospatial functioning. In relation to mood disorder, evidence relating to both pharmacological and psychological therapies for depression is currently inconclusive and so no firm recommendations were made. For anxiety there was sufficient evidence to recommend cognitive behaviour therapy be considered. Conclusions: The guideline development process highlights that the evidence base in relation to rehabilitation of cognitive impairment is now sufficiently well developed (albeit still limited) to warrant some specific recommendations. However the evidence base in relation to management of mood disorder remains largely insufficient upon which to base clear recommendations. Evidence for the effectiveness of comprehensive (holistic) neuropsychological rehabilitation programmes is present, though studies of such programmes cannot identify active ingredients. We will discuss future research priorities necessary for the development of clinically useful guidelines.

Correspondence: Jonathan Evans; jonathan.evans@glasgow.ac.uk 


\title{
Cognitive Behavioral Therapy for Depression Following Traumatic Brain Injury: Findings of a Randomized Controlled Trial
}

\author{
Ashman, Teresa ${ }^{1}$ and Tsaousides, Theodore ${ }^{2}$ \\ ${ }^{1}$ Rusk Institute of Rehabilitation Medicine, New York University Langone Medical Center, New York, USA \\ ${ }^{2}$ Department of Rehabilitation Medicine, Mount Sinai School of Medicine, New York, USA
}

\begin{abstract}
$B$ ackground and aims: Few published studies (mostly case reports, case series, and uncontrolled single-case design studies) exist evaluating the effectiveness of psychotherapy for post-traumatic brain injury (TBI) depression and findings have been mixed. Given the high prevalence and negative impact of post-TBI depression, developing appropriate behavioral interventions is imperative. Cognitive-behavioral therapy (CBT) is a well-established treatment for depression in the general population. The structure and frame of CBT render it a suitable treatment for individuals with TBI-related cognitive challenges. However, the efficacy of CBT in post-TBI depression has not been investigated. The purpose of this presentation is to describe the outcomes of a recently completed randomized controlled trial of the efficacy of psychotherapy for post-TBI depression. Method: A study was designed to compare the efficacy of CBT and supportive psychotherapy (SPT) in treating depression following TBI. Participants included individuals with TBI who met DSM-IVTR criteria for depressive disorder. They were randomly assigned to either CBT or SPT and received 16 psychotherapy sessions. Participants' mood (diagnosis and symptom severity) and psychosocial functioning were assessed at four time points (pre-treatment, post-session 9, post-treatment, 6-month follow-up). Results: Forty-one participants completed treatment and followup assessments $(\mathrm{CBT}=21, \mathrm{SPT}=20)$. Approximately $57 \%$ of the CBT and $35 \%$ of the SPT group no longer had a DSM-IV-TR depressive disorder diagnosis, representing significant change for both groups $(p=.00004$ and $p=.003$, respectively). Rate of remission did not differ significantly between groups $(p=.134)$. Conclusions: The study provides evidence that individual psychotherapy is an effective treatment for post-TBI depression. The structure and techniques provided by CBT render it a preferred treatment when working with individuals with cognitive impairments. Implications of these findings for practice and research are discussed.
\end{abstract}

Correspondence: Teresa Ashman; teresa.ashman@nyumc.org

\section{SESSION 6: PAEDIATRIC ASSESSMENT AND REHABILITATION}

\section{Post-traumatic amnesia in children: Natural sequence of recovery}

Briggs, Rachel H. ${ }^{1}$; Birse, Jason ${ }^{2}$; Tate, Robyn ${ }^{3}$; Epps, Adrienne ${ }^{2}$; Brookes, Naomi ${ }^{2}$ and Lah, Suncica ${ }^{1}$

${ }^{1}$ School of Psychology, University of Sydney, Sydney, Australia

${ }^{2}$ Brain Injury Rehabilitation Program, Sydney Children's Hospital (Randwick), Sydney, Australia

${ }^{3}$ Rehabilitation Studies Unit, Sydney Medical School, University of Sydney, Sydney, Australia

\begin{abstract}
$B$ ackground and aims: Post traumatic amnesia (PTA), which arises following traumatic brain injury (TBI), is characterised by global cognitive dysfunction, including disorientation and amnesia. Although PTA is routinely assessed in children, no published study has examined (i) natural sequence of PTA recovery in children, and (ii) the relations between chronological age and PTA resolution. Method: Between September 2007 and November 2010 ninety seven children (aged 8 to 15 years) were admitted to the Brain Injury
\end{abstract}


Rehabilitation Program at Sydney Children's Hospital (Randwick). All children were administered the Westmead PTA Scale (WPTAS), which assesses orientation (to person, time and place) and memory (for examiner and pictures). Scores of children with PTA $>24$ hours $(n=44)$ were examined. Results: Mean duration of PTA was 5.4 days $(\mathrm{SD}=4.92)$. Orientation was regained significantly before memory $(\mathrm{t}(42)=3.08, \mathrm{p}<.01)$. When regaining orientation, children become oriented for person first, place second and time last. Orientation to time was regained significantly later than orientation to person and place, $\mathrm{F}(2,84)=16.72, \mathrm{p}=0.001$. When re-gaining memory, children remembered the examiner first and pictures second; but the difference between memory for the examiner compared to pictures was not significant, $\mathrm{F}(1,43)=2.49, \mathrm{p}=0.12$. Correlations between chronological age and days taken to regain orientation and memory were not significant. Conclusions: Our study suggests that in school-aged children who are in PTA amnesia resolves after disorientation. Importantly, duration of disorientation and amnesia was not related to chronological age, which confirms that the WPTAS is a valid measure of orientation and memory in this patient population.

Correspondence: Suncica Lah; suncica.lah@sydney.edu.au

\section{Towards a novel ecologically-valid assessment of executive functions in children and adolescents: Could virtual reality be the answer?}

Jansari, Ashok ${ }^{1}$; Edmonds, Caroline ${ }^{1}$; Gordon, Rebecca ${ }^{1}$; Nwosu, Ukachi ${ }^{1}$ and Leadbetter, Tony ${ }^{1}$

${ }^{7}$ School of Psychology, University of East London, London, UK

$B$ ackground and aims: Childhood and adolescence is a time of rapid cognitive change. As the prefrontal cortex expands, associated improvements in executive functions are especially marked. Most executive function assessment is laboratory based. JAAM ${ }^{\complement}$ provides a novel ecologically-valid assessment of executive functions in adults using virtual reality to assess impacts of brain injury (Jansari et al, 2004). We aimed to develop and evaluate a children's version, (JAAM- ${ }^{\complement}$ ) to assess executive function in children and adolescents. Methods: JAAM-C ${ }^{\complement}$ was designed to assess eight cognitive constructs; planning, prioritisation, selection, creative-thinking, adaptive-thinking, action-based prospective memory (PM), event-based PM and time-based PM. Resembling a computer game, participants roleplay running their own birthday party. Thirty typically-developing children aged 10-12 (5F, 7M), 13-15 (3F, $6 \mathrm{M})$ and 16-18 (5F, 4M) completed JAAM-C ${ }^{\complement}$. Results: Significant positive correlations of age with prioritisation, event and time based PM and total score were found. Further, a one-way MANOVA on performance revealed a significant main effect of age (Wilks' $\lambda=.151, \mathrm{~F}(18,38)=3.3, \mathrm{p}<.005)$. Therefore, JAAM-C $C^{\complement}$ demonstrated a robust ability to accurately identify differences in individual cognitive constructs and overall executive function as a function of age during adolescence. Discussion and Conclusions: JAAM-C ${ }^{\complement}$ offers an assessment of executive function and its developmental trajectory providing a profile across a range of cognitive constructs that current assessments cannot. Further, it is the first ecologically valid tool for assessing executive functions in childhood and adolescence. There is potential to use JAAM-C as an assessment tool for typical and atypical development or executive dysfunction due to traumatic brain injury.

Correspondence: Ashok Jansari; a.jansari@uel.ac.uk 


\title{
Paediatric goal management training for children with severe traumatic brain injury (TBI)
}

\author{
Krasny-Pacini; Agata ${ }^{1,2}$; Limond, Jenny ${ }^{3}$; Evans, Jon ${ }^{3}$ and Chevignard, Mathilde ${ }^{2,4}$ \\ ${ }^{7}$ Paediatric Rehabilitation Department, Clémenceau Rehabilitation Centre and Hautepierre University Hospital, \\ Strasbourg, France \\ ${ }^{2}$ Laboratory ER - 6, Pierre and Marie Curie University - Paris 6, Paris, France \\ ${ }^{3}$ Academic Unit of Mental Health and Wellbeing; Institute of Health and Wellbeing; University of Glasgow; \\ Gartnavel Royal Hospital; Glasgow; UK \\ ${ }^{4}$ Rehabilitation Department for Children with Acquired Brain Injury, Saint Maurice Hospital, Saint Maurice, \\ France
}

\begin{abstract}
$B$ ackground and Aims: Goal management training is a metacognitive training of executive functioning used in adults, and it has recently been adapted for children (pGMT). The aims of this pilot study were to: i) assess effectiveness of pGMT; ii) examine the participation of the child's 'everyday people' (parent, teacher and school assistant) in cognitive rehabilitation; and iii) explore factors that influence engagement and outcome. Methods: A single case experimental design with multiple-baselines was used. Four children aged 9-14 years, who had experienced a severe brain injury, were included. Assessments were administered twice prior to intervention (baseline), and immediately post-intervention, and at 3- and 6-month follow-ups. Participants received fifteen weekly one-hour individual sessions of pGMT. To promote generalisation and transfer, practical applications were included and the 'everyday people' were provided with session content and applications for school and home. Results: Participants improved either on the BRIEF or on the Children's Cooking Task. All showed progress towards their goals, although none reached 'expected' outcome (GAS). Everyday people's participation was low, especially at school. Generalisation and transfer were poor, except for one child who had paradoxically poor self-awareness. Conclusion: Paediatric GMT is a feasible and promising method for improving daily executive functioning. Its effortful nature, however, makes pGMT difficult to apply throughout the child's day, possibly contributing to the limited generalisation effects. Individual differences in outcomes will be discussed in relation to neurocognitive, emotional, and psychosocial characteristics. Potential further adaptations to pGMT to aid transfer and participation, and our future research, will also be discussed.
\end{abstract}

Correspondence: Agata Krasny-Pacini; agatakrasny@yahoo.com

\section{Prospective memory intervention for adolescents with acquired brain injury: De- velopmental and psychosocial factors affecting outcomes}

\footnotetext{
Rous, Rebecca'; Adams, Malcolm'; Fish, Jessica²; Manly, Tom² and Adlam, Anna ${ }^{1,3}$

${ }^{7}$ Department of Psychological Sciences, Norwich Medical School, University of East Anglia, Norwich, UK

${ }^{2}$ Cognition and Brain Sciences Unit, Medical Research Council, Cambridge, UK

${ }^{3}$ Cambridge Centre for Paediatric Neuropsychological Rehabilitation, Cambridge and Peterborough Foundation Trust, Cambridge, UK
}

ackground and aims: Prospective memory (PM) problems are common
after paediatric acquired brain injury (ABI). Emerging evidence from the
adult literature supports the use of strategies targeting the executive functioning
aspects of PM. Building on work by Fish et al. (2007), this study aimed to adapt
and pilot an intervention for adolescents with PM difficulties following ABI.
Method: A single-case series design with randomised alternating treatments 
was used. Seven adolescents (12-17 years) with ABI completed a PM task which involved making three phone calls per day at specific times for 3 weeks. After one week of calls brief goal management training (GMT), teaching the mnemonic 'STOP' (Stop, Think, Organise, Plan) to cue a mental review of goals, was provided. Six text messages reading 'STOP' were then sent to participants' mobile phones at random times on 5 of the 10 following working days. Ordering of text message days was also randomly selected. Results: At a group level, performance (number and accuracy of phone calls) was significantly better on cued days. At the individual level, significant effects of cueing was found for four of the seven cases. Five participants showed transfer effects, reporting gains in real-life PM achievements. Conclusions: The intervention shows promise for adolescents with ABI. Neurocognitive, emotional, and psychosocial characteristics will be considered in relation to individual performance. Future research, in terms of evaluating generalisability on a larger scale and promoting attainment of real-life PM goals in adolescents following brain injury, will also be discussed.

Correspondence: Anna Adlam; a.adlam@uea.ac.uk

\section{POSTERS - MONDAY - Assessment and conceptualisation in rehabilitation}

\section{Psychological Outcome in Association with a Lack of Congruency in Patient/Carer Perspectives in Brain Tumour Patients Following Surgery: Implications for Therapy}

Andrewes, Holly E. ; Drummond, Kate ${ }^{2}$; Rosenthal, Mark ${ }^{2}$; Bucknill, Andrew ${ }^{3}$ and Andrewes, David G. ${ }^{1}$

${ }^{1}$ Department of Psychological Sciences, University of Melbourne, Australia

${ }^{2}$ Department of Neurosurgery, Royal Melbourne Hospital, Australia

${ }^{3}$ Department of Orthopaedic Surgery, Royal Melbourne Hospital, Australia

ackground and aims: Brain tumour patients following surgery have been
shown to experience personality changes and emotional dyscontrol. This
can alter the patient's relationships, and contribute to caregiver distress. Re-
search with terminally-ill patients has shown that carers are more pessimistic
than patients about such psychological changes (Field et al., 1995). This study
investigated if such a disparity exists for tumour patients (TP) with poor prog-
nosis following neurosurgery. Method: 33 post-surgery TP and carers (exper-
imental group), and 29 post-surgery orthopaedic patients and carers (control
group) completed a survey investigating changes in the patient's emotions,
relationships, coping and psychological well-being (Brain Injury Community
Rehabilitation Outcome scale), and patient/carer anxiety and depression lev-
els (Hospital Anxiety and Depression Scale). Results: Carers of TPs rated the
patients'psychological wellbeing as significantly worse than the patients' self-
ratings. Carers of TPs were also significantly more likely than TPs to perceive
changes in the patient's emotions, relationships and coping skills as causing
problems. This disparity was not found in the control group. Anxiety was sig-
nificantly higher for carers in both groups, yet only positively correlated with
the disparity in ratings of the patients' psychological well-being and changes
in relationship status in the experimental group. Conclusions: Carers of ma-
lignant tumour patients following neurosurgery are more pessimistic about
the patient's psychological problems and relationship, and this discrepancy is
associated with increased anxiety. The results are discussed in terms of how 
the lack of congruency between the TPs' and carers' perceptions of the patient might be gainfully addressed within the context of interpersonal therapy.

Correspondence: Holly Andrewes; h.andrewes@ hotmail.com

\title{
Dissociation between words and number words: a case study of traumatic brain injury
}

\author{
Baird, Amee ${ }^{1}$ and Robinson, Gail ${ }^{2}$ \\ ${ }^{1}$ Hunter Brain Injury Service, Newcastle, Australia \\ ${ }^{2}$ School of Psychology, The University of Queensland, Brisbane, Australia
}

\begin{abstract}
$B$ ackground and aims: Case studies have shown that processing of Arabic numerals (e.g. 9) and number words (e.g. nine) can be selectively impaired or preserved, suggesting that these two types of words are processed separately by the language system. The study aim was to examine word and number word processing skills in a man (NB) with severe global aphasia and relatively preserved numerical cognition following a severe traumatic brain injury. Method: We designed an experimental lexical decision task, the $\mathrm{Nu}-$ merical Orthographic Lexical Retrieval Task (NOFT) and a range of brief experimental tasks to explore NB's ability to gesture numerical information. Results: NB's performance on the NOFT was errorless (25/25), but on a verbal equivalent his performance was essentially at chance (27/50). This finding indicates that he had preserved ability to process lexical items that contained number words (e.g. nine) but impaired processing of non-number words (irregular or archaic). His ability to produce and understand finger gestures representing numerical information was relatively well preserved in the written modality compared with the oral modality. Conclusion: This case study contributes to the few published case studies to date that show that words and number words are mediated by different cognitive mechanisms. In addition, it provides preliminary evidence for a dissociation between number and action gestures. To the best of our knowledge there has been no previous investigation of number versus non-number gesture production or interpretation. These findings have implications for the use of gesture in rehabilitation of aphasia.
\end{abstract}

Correspondence: Amee Baird; ameebaird@gmail.com

\section{Introducing Baseline Neuropsychological Assessment for Children Diagnosed with a Brain Tumour: Reflections and Case Examples a Year on}

\author{
Bennett, Emily ${ }^{1}$; Wilne, Sophie ${ }^{2}$; Talbot, Emily ${ }^{1}$ and Thomas, Sophie ${ }^{1}$ \\ ${ }^{1}$ Department of Clinical Psychology and Neuropsychology, Queen's Medical Centre, Nottingham University \\ Hospitals NHS Trust, Nottingham, UK. \\ ${ }^{2}$ Department of Paediatric Oncology, Queen's Medical Centre, Nottingham University Hospitals NHS Trust, \\ Nottingham, UK.
}

$B$ ackground and aims: To explore issues relating to the introduction of a new neuropsychological care pathway for children and adolescents diagnosed with a brain tumour at a large regional centre in the UK. Method: The care pathway includes the administration of a brief baseline neuropsychological assessment after diagnosis, ideally prior to surgery and treatment. Children and adolescents are then followed-up for a more detailed assessment one year after diagnosis and at key transition points after this. Results: The experiences of the team in the first year of completing the baseline assessments are 
discussed. This includes the key challenges, particularly with regard to practical and emotional issues, and the main benefits for both patients and the multi-disciplinary team. The validity of baseline assessments is also considered in relation to the content and focus of the assessment battery used. These issues are illustrated by two case examples of children assessed at baseline, who have now been followed up for a neuropsychological assessment one year after diagnosis. Conclusions: After the first year of the new care pathway, the overall benefits of baseline neuropsychological assessment have become apparent. Despite challenges, baseline assessments have offered useful information for the team, families and schools, and also ensure that the Trust's psychologists are involved with patients from the outset. This offers opportunity for more comprehensive emotional, cognitive and behavioural support that is tailored to the individual child's needs.

Correspondence: Emily Bennett; Emily.bennett@nuh.nhs.uk

\title{
Ecological validity and reliability of a neuropsychological test measuring executive functioning
}

\author{
Bertens, Dirk ; Boelen, Danielle ${ }^{1}$; Fasotti, Luciano ${ }^{1}$ and Kessels, Roy PC ${ }^{1,2}$ \\ ${ }^{1}$ Donders Institute for Brain, Cognition and Behaviour, Radboud University Nijmegen, Nijmegen, The Nether- \\ lands \\ ${ }^{2}$ Department of Medical Psychology, Radboud University Nijmegen Medical Centre, Nijmegen, The Nether- \\ lands
}

\begin{abstract}
$B$ ackground and aims: Ecologically valid tasks such as the Modified Six Elements Test (MSET) are widely used to assess executive impairments. However, its predictive value for everyday executive function is unclear. Furthermore, interpreting changes over time could be influenced by learning effects. The objective of this study is to examine ecological validity, test-retest reliability and parallel-forms reliability of two newly designed parallel forms of the MSET to contribute to a better assessment of executive functioning of patients in neuropsychological rehabilitation. Methods: In this study both parallel forms of the MSET will be administered in healthy adults with a 1-2 week interval. To examine executive functioning in daily life the Executive Function Index (EFI-NL) self-report questionnaire will be administered. MSET's ecological validity will be examined by correlating its test scores with the EFI-NL. Both the test-retest reliability and the parallel-forms reliability will be evaluated by calculating smallest detectable differences (SDD). Results: So far 30 participants have enrolled in the study (8 males, mean age: 31.6, $S D$ : 13.3; mean estimated IQ: $108.3, S D=4.3)$. Results show a moderate correlation between the MSET scores and the EFI-NL $(r=.34)$. Test-retest reliability was adequate $(p=.318, \mathrm{SDD}=26.61)$. Parallel-forms reliability was sufficient $(p=.035, \mathrm{SDD}=24.97)$. None of the participants crossed the SDDs. Conclusions: The present study shows that self-reported executive functioning in healthy adults correlates with the performance on a complex planning task, thus supporting the ecological validity of the MSET. Test-retest reliability and parallel-forms reliability were considered adequate for measuring executive functioning over time. These psychometric properties need to be examined for patients with brain injury.
\end{abstract}

Correspondence: Dirk Bertens; d.bertens@donders.ru.nl 


\title{
A tool for teaching case planning in neuropsychological rehabilitation: The Hy- potheses Table
}

\author{
Bolognani, Silvia Adriana Prado ${ }^{1}$ and Bueno, Orlando Francisco Amodeo ${ }^{1,2}$ \\ ${ }^{1}$ Centro Paulista de Neuropsicologia, Associacao Fundo de Incentivo a Pesquisa, Sao Paulo, Brazil \\ ${ }^{2}$ Departamento de Psicobiologia, Universidade Federal de Sao Paulo, Brazil
}

\begin{abstract}
$B$ ackground and aims: In Brazil, professional training in neuropsychology is optional for psychologists. Professionals are fully licensed for clinical practice after graduation and most of them are never taught about cognitive functions while at university. Nationally accredited post-graduation courses mostly educate professionals on cognitive functions, tests and assessment, with little or no study or training in rehabilitation. Admission interviews with candidates show that a great number of professionals applying for courses in neuropsychological rehabilitation are actually searching for cognitive training tools, with the belief that restoration of lost functions can be trained with test-like material or "brain-buster" software. Thus, motivating students to take a more effective but "less easy road" has been a great challenge for groups trying to train professionals on non-restorative approaches and evidence-based rehabilitation. Method: Here we present a simple tool created to help the beginner clinician to organise a rehabilitation plan, select "SMART" goals and adequate intervention techniques, based on a holistic understanding of brain injury cases.

The tool was created based on the cognitive therapy framework, where the use of diagrams and templates play an important role in intervention planning. Results: Although a controlled study is yet to be conducted using this tool, the Hypotheses Table has proven helpful in our daily clinical practice, and most importantly, in helping students understand there is much more to rehabilitation than cognitive training. Conclusion: Experience in using the Hypotheses Table suggests that professional trainees benefit from this approach to case planning in neuropsychological rehabilitation.
\end{abstract}

Correspondence: Silvia Adriana Prado Bolognani; silvia.bolognani@gmail.com

\section{The dynamic assessment approach in persons with cognitive impairments: a sys- tematic review}

Boosman, Hileen'; Bovend'Eerdt, Thamar²; Visser-Meily, Johanna ${ }^{1}$ and Van Heugten, Caroline ${ }^{3}$

${ }^{1}$ Rudolf Magnus Institute of Neuroscience and Center of Excellence for Rehabilitation Medicine, University Medical Center Utrecht and De Hoogstraat, Utrecht, The Netherlands

${ }^{2}$ Department of Human Movement Sciences, Maastricht University, Maastricht, The Netherlands

${ }^{3}$ Maastricht University Medical Centre, Department of Psychiatry and Neuropsychology, School for Mental Health and Neuroscience and Maastricht University, Department of Neuropsychology and Psychopharmacology, Maastricht, The Netherlands

$\boldsymbol{B}$ ackground and aims: The potential to learn can be determined through a dynamic assessment (DA) procedure. The prime aim of DA is to determine the extent to which performance improves when guidance is given. Currently, DA is rarely employed in acquired brain injury (ABI) rehabilitation. The objective of this study was to evaluate the clinical usefulness of DA in terms of learning potential classification and outcome prediction in persons with cognitive impairments. Method: A systematic literature search on DA in persons with cognitive impairments was conducted. Three electronic databases 
were searched: PubMed, PsychINFO and Embase/Medline. Two authors independently reviewed the articles. Results: In total, 27 articles were included. Most studies $(n=19)$ included persons with schizophrenia or schizoaffective disorder. Only three studies included persons with ABI. The most frequently used DA procedure was the dynamic Wisconsin Card Sorting Test (WCST) $(n=16)$. The methodology used to classify patients' learning potential varied from the calculation of gain scores to categorizing patients using algorithms (e.g. non-learner, learner, high-achiever). Furthermore, seven out of nine longitudinal studies reported a high predictive validity of the DA. Conclusions: There are promising results regarding the predictive value of learning potential in persons with cognitive impairments. The implications for clinical practice and future research will be discussed.

Correspondence: Hileen Boosman; h.boosman@dehoogstraat.nl

\section{Dynamic assessment: is it useful for assessing learning potential in patients with $A B I$ ? Preliminary results of an ongoing study}

Boosman, Hileen ${ }^{1}$; Visser-Meily, Johanna ${ }^{1}$; Nassime, Sabrine ${ }^{2}$ and van Heugten, Caroline ${ }^{2}$

${ }^{7}$ Rudolf Magnus Institute of Neuroscience and Center of Excellence for Rehabilitation Medicine, University Medical Center Utrecht and De Hoogstraat, Utrecht, The Netherlands

${ }^{2}$ Maastricht University, Department of Psychiatry and Neuropsychology, School for Mental Health and Neuroscience and Maastricht University, Department of Neuropsychology and Psychopharmacology, Maastricht, The Netherlands

$B$ ackground and aims: Classifying a patient's learning potential may facilitate the choice of appropriate rehabilitation. Dynamic assessment (DA) procedures aim to determine the extent to which performance improves when guidance is given. The dynamic Wisconsin Card Sorting Test (WCST) is a frequently used DA but is rarely used for this purpose after acquired brain injury (ABI). This study aimed to evaluate the use of the dynamic WCST in inpatient rehabilitation. Method: Patients with ABI were administered three consecutive administrations of the WCST in a pre-post training design. The pre-test and post-test followed standard WCST procedures and during the training phase additional instructions and feedback were given. Learning potential was evaluated by comparing pre- and post-test performance. Results: To date, the dynamic WCST has been administered to $10 \mathrm{ABI}$ patients. Five patients showed constantly normal performance. Four patients had impaired performance $(\geq-1.5 \mathrm{SD})$ on the pre-test with a high number of perseverative errors $(\geq-1.5 \mathrm{SD})$. For three of these patients performance was in the normal range after training and for one patient performance did not change. One patient had adequate performance at the pre-test but demonstrated a major decrease in performance post-test. Conclusions: These preliminary results suggest that persons with impaired performance on the WCST differ in the extent to which they benefit from guidance.

Correspondence: Hileen Boosman; h.boosman@dehoogstraat.nl 


\title{
Personal construction of self before and after a rehabilitation program
}

Covre, Priscila ${ }^{1,2}$; Bolognani, Silvia Adriana Prado ${ }^{2}$; Drumond, Debora ${ }^{2}$; Landucci, Daniella ${ }^{2}$; Rodrigues, Anair ${ }^{2}$; Monteiro, Maria de Fátima ${ }^{1,2}$; Queiroz, Fernanda Pamplona de ${ }^{2}$; Martins, Marjorie Regina ${ }^{1,2}$; Simonetti, Luciane ${ }^{2}$ and Bueno, Orlando Francisco Amodeo ${ }^{1,2}$

${ }^{1}$ Departamento de Psicobiologia; Universidade Federal de Sao Paulo; Sao Paulo; Brazil

${ }^{2}$ Centro Paulista de Neuropsicologia; Associaçao Fundo de Incentivo a Pesquisa; Sao Paulo; Sao Paulo; Brazil

\begin{abstract}
$B$ ackground and aims: This study sought to investigate how people make sense of themselves after a holistic rehabilitation program. Methods: Ten individuals who had experienced acquired brain injury engaged in small group exercises based on a personal construct approach. Bipolar constructs were elicited at two phases of the rehabilitation program: (1) at the beginning of the program, through systematic comparison of pre-injury, current and ideal selves; and (2) at the end of the program, through comparison of selves before and after rehabilitation. Constructs elicited in both situations were subjected to a thematic analysis based on the nine themes proposed by Gracey, Palmer, Rous et al. (2008). Results: The highest proportion of constructs comparing pre and post injury selves fell into the following themes: "basic skills" (31.5\%), "experience of self in relation to self" (21\%), "experience of self in the world" (18.4\%) and "emotions" $(10.5 \%)$. When comparing their selves before and after rehabilitation, theme distribution was slightly different: "basic skills" (40\%), "experience of self in the world" (32\%), "experience of self in relation to self" (12\%) and "outlook/coping" (8\%). Conclusion: Patients' self-construal after rehabilitation corroborates clinical impressions that individuals were more aware of their deficits and capacities and of their active role in the world.
\end{abstract}

Correspondence: Priscila Covre; priscilacovre@gmail.com;

\section{The role of premorbid psychological processes in outcomes following bilateral thalamic infarction: Two case examples}

Crawford, Linda $^{1}$; Berzengi, Azi ${ }^{1}$; Sumners, David ${ }^{1}$; Humphries, Clare ${ }^{1}$; Shorto, Gabriella ${ }^{1}$; Collins, Laura'; Dybiec, Sarah"; Moffat, Claire ${ }^{1}$; Massingham, Lee ${ }^{1}$; Bowers, Louise ${ }^{1}$ and Bailey, Michelle ${ }^{1}$

${ }^{1}$ The Brain Injury Rehabilitation Trust (BIRT, Fen House, Ely, UK

$B$ ackground and aims: The impact of bilateral thalamic infarction, sustained in adulthood, on autobiographical memory has been well documented. The aim of this study was to identify the role of premorbid psychological processes (e.g., cognitive and behavioural coping styles) in outcomes following injury and treatment planning. Method: Two individuals (aged 56 and 65 years) with bilateral thalamic infarction were admitted to a residential brain injury rehabilitation assessment and treatment programme. Both participants completed a thorough multi-disciplinary assessment including neuropsychology and neuropsychiatry. Interviews with the participant's partner ascertained major life events, pre-morbid personality, and coping characteristics. Results: The neurocognitive profiles of both individuals were consistent with the literature, however, the two individuals differed in terms of premorbid major life events, personality, and coping characteristics. Case 1 had a history of attempted suicide and domestic violence with no documented psychiatric diagnosis. Neurobehavioural features of anxiety, confabulation, and 
reassurance seeking were dominated by perceived threat of violence and formed the focus of treatment. Case 2 displayed coping styles consistent with premorbid coping; however, these were exaggerated post-injury (e.g., social isolation, self-criticism) and formed potential barriers to treatment. The rehabilitation approach for Case 2, therefore, also focused on coping styles. Conclusions: Two individuals with similar neuroanatomical diagnoses and neurocognitive profiles presented with different neurobehavioural difficulties. The neurobehavioural features became the focus of treatment and discharge planning due to their impact on engagement in rehabilitation and longer-term outcome. These cases highlight the need to consider premorbid psychological processes when planning funding and treatment needs of individuals with bilateral thalamic infarctions.

Correspondence: Linda Crawford; linda.crawford@thedtgroup.org

\section{Facilitating senior rehabilitation support workers to think reflectively improves their clinical practice and confidence}

Crawford, Linda ${ }^{1}$; Collins, Laura ${ }^{1}$ and Massingham, Lee ${ }^{1}$

${ }^{1}$ The Brain Injury Rehabilitation Trust (BIRT), Fen House, Ely, UK

$B$ ackground and Aims: Rehabilitation support workers (RSWs) in the residential rehabilitation setting are pivotal in supporting the consolidation of therapy interventions. The process and effectiveness of sharing psychological formulations is under-researched, however, some studies indicate that this can be helpful. This study aimed to evaluate whether facilitating RSWs in psychological formulation and reflective thinking skills helped to increase their understanding of the service user. Method: Six RSWs were supervised in pairs once every six weeks by the Consultant Clinical Psychologist, Senior Occupational Therapist, or Senior Neuro-Physiotherapist. RSWs rated their work satisfaction, skill, mood, understanding of the service user formulation, and confidence, prior to supervision. During supervision, the therapist and RSWs developed a shared formulation of the service user's difficulties. Events involving the RSW and service user were then discussed in relation to the formulation, using reflective dialogue. To promote consolidation of formulation skills outside of supervision, participating RSWs were asked to share the formulation with another member of the team. Outcomes were evaluated by re-administering the baseline measures. Results: Senior RSWs rated their understanding and confidence of the service user formulation more highly following the intervention. Conclusions: Sharing the psychological formulation and supporting reflective thinking was beneficial in terms of increasing understanding and confidence of the senior RSWs working with the service user. Recommendations for future research further evaluating the use of psychological supervision, particularly in terms of service user experience and rehabilitation outcomes, will be discussed.

Correspondence: Linda Crawford; linda.crawford@ thedtgroup.org 


\title{
Feasibility of single-case study designs to evaluate neuropsychiatric treatment of behaviour and mood disorders after severe acquired brain injury
}

Eilander, Henk ${ }^{1}$; ter Mors, Bert ${ }^{1}$; Franssen, Frans ${ }^{1}$; Heutink, Matagne ${ }^{1}$; van Heugten, Caroline $^{2,3}$ and Ponds, Rudolf ${ }^{2}$

${ }^{7}$ Department of Brain Injury, Huize Padua, GGZ Oost Brabant, Boekel, the Netherlands

${ }^{2}$ School for Mental Health \& Neuroscience, Maastricht University, Maastricht, the Netherlands

${ }^{3}$ Department of Neuropsychology \& Psychopharmacology, Maastricht University, Maastricht, the Netherlands

\begin{abstract}
$B$ ackground and aims: Neuropsychiatric treatment of behaviour or mood disorders after severe acquired brain injury generally consists of pharmacological interventions combined with behavioural treatments. Systematic research on the effects is scarce, due to methodological and practical problems. We used the criteria for Single-Case Experimental Designs developed by Tate et al. (2008) to investigate the feasibility of single-case studies on the effects of psychopharmalogical interventions in a neuropsychiatric setting. The results of ten patients are reported. Method: In non-selected cases, which were subject to psychopharmalogical treatment, a naturalistic $\mathrm{AB}(\mathrm{A})(\mathrm{B})$ research design was developed. A multiple set of measurements was tailored individually, based on the specific behaviour that was targeted for intervention. Results: The study was executed in ten patients. Three patients suffered from a mood disorder, three from apathy, and four from disruptive behaviour. Twelve different pharmacological agents were involved. In five cases, multiple agents were prescribed or cut back. Evaluative instruments were: tests or tasks, observation by staff members, Visual Analogue Scales or Likert-scales and questionnaires administered to patients or a family member. The case studies differed substantially in content and length. We will present detailed data from the studies on methylphenidate (4 patients) and amantadine ( 2 patients). Conclusions: Although case studies are feasible in a clinical setting with limited resources, the naturalistic character of this study resulted in too much variability to be able to draw firm conclusions. Therefore, application of experimental designs is considered inevitable.
\end{abstract}

Correspondence: Henk Eilander; h.j.eilander@brain-consult.nl

\section{Back to work after stroke. A qualitative study focusing on experiences of getting back to work after stoke}

\author{
Einbu, Guri ${ }^{1}$ \\ ${ }^{7}$ Department of Rehabilitation, Innlandet Hospital Trust, Norway
}

$B$ ackground and aims: The aim of this study was to get descriptions of experiences from a rehabilitation process in terms of getting back to work after stroke, and how this process was affected by participating in "Raskere tilbake", a return to work project. Method: A qualitative method with semistructured interviews was used to collect data from three respondents who have had a stroke. The study applied both an occupational perspective (Kielhofner et al, 1997) and an interactional scientific perspective (Goffman, 1959). An interactional perspective together with Erving Goffman's dramaturgical model (1959) has been used to analyze the material. These perspectives both focus on the interaction between humans and the environment, and constitute a ground for the understanding of the common, yet interesting events in people's rehabilitation processes. The interviews were reviewed several times and words that could describe the themes were noted. In the process of further analysis, 
patterns with several subcategories were explored that eventually formed the two main dimensions of findings. Results: The findings of this study are related to two main dimensions: (1) Individual efforts in the rehabilitation process of returning to work after stroke; and (2) Teamwork in the rehabilitation process of returning to work after stroke. The main dimensions contain several subcategories. Individual efforts consist of exercises in order to identify, improve and keep up the body's functional level. Respondents had to get an overview of their own resources, find out how to live their lives with a lack of energy, and they had to figure out how to present themselves and adapt to a new social role after the stroke. The teamwork dimension consists of three subcategories of teams: A team with colleagues and leaders at work, a team with peers in the "Raskere tilbake"-project, and a team with staff from the "Raskere tilbake"project. Conclusion: Teamwork strengthened individuals in their own efforts and was an important contribution in the rehabilitation process of returning to work after stroke.

Correspondence: Guri Einbu; Guri.Einbu@sykehuset-innlandet.no

\title{
Who needs what and how do we know? Capturing complexity in provision of ser- vices for adults and children with Acquired Brain Injury (ABI)
}

\author{
Gracey, Fergus ${ }^{1,3,4}$; Malley, Donna ${ }^{1,3} ;$ Komashie, Alex ${ }^{2,3}$ and Bateman, Andrew ${ }^{1,3}$ \\ ${ }^{1}$ Oliver Zangwill Centre for Neuropsychological Rehabilitation, Cambridgeshire Community Services NHS \\ Trust, Cambridgeshire, UK \\ ${ }^{2}$ Engineering Design Centre, Universitry of Cambridge Dept of Engineering, Cambridge, UK \\ ${ }^{3}$ NIHR Collaborations for Leadership in Applied Healthcare and Research for Cambridgeshire and Peterbor- \\ ough, Cambridgeshire and Peterborough NHS Foundation Trust, Douglas House, Cambridge, UK. \\ ${ }^{3}$ Cambridge Centre for Paediatric neuropsychological Rehabilitation, Cambruidgeshire and Peterborough \\ NHS Foundation Trust, Heron Court, Cambridge, UK.
}

ackground and aims: Tools that facilitate service planning and evalua-
tion are imperative in the context of economic challenges to healthcare
delivery. Recent publications have contributed to the systematisation of out-
come measurement in rehabilitation. However, challenges remain with regard
to capturing complexity of needs. We report on work to develop tools that
may support service provision and evaluation for people with complex or
'hidden disability' post ABI. Method: The following processes were under-
taken: policy analysis of selected documents that guide practice, review of
approaches to assessing complexity of needs in rehabilitation, and character-
isation of accepted referals to specialised neuropsychological rehabilitation
centres for adults ( $n=14)$ and children ( $n=22)$ over a fixed time period. Re-
sults: Documents that guide practice highlight the presence of complex prob-
lems following brain injury but definition of the term and guidance regarding
intervention is lacking. Conceptualisation of complexity in rehabilitation fo-
cuses on level of need and amount or intensity of input. Complexity as defined
in systems engineering and management provides additional constructs that
allow consideration of interactions and relationships between the intra and
interpersonal aspects. Case data illustrates adults and children with multiple
pre- and post-injury difficulties for whom established complexity assessments
may not be sensitive. Conclusion: A systems approach to the conceptualisation
of complexity suggests two dimensions of measurement that may be helpful:
'certainty of causal relationships' and number of differing perspectives regard-
ing nature of difficulties and needs. These may prove helpful in identifying 
appropriate resources for rehabilitation. A 'stepped care process' to support triaging for rehabilitation according to this conceptualisation is provided.

Correspondence: Fergus Gracey; fg290@medschl.cam.ac.uk

\title{
Neuropsychological diagnostics and speech therapy method selection
}

\author{
Khrakovskaya, Maria ${ }^{1}$ \\ ${ }^{1}$ Institute of the Human Brain, Russian Academy of Sciences, Saint-Petersburg, 194356 Box. 9, Russia
}

\begin{abstract}
$B$ ackground and aims: This study aimed to compare the utility of two rehabilitation therapy methods: the Vygotsky-Luria neuropsychological approach and cognitive language system-based models (Basso, 1993). The concept of a complex brain-systems network underlies the neuropsychological approach whereby a pattern of different language deficits occur following selective damage to specific areas of the network. Method: BA was a 42 year old man with focal left hemisphere lesions and a fluent aphasia that had shown no substantial improvement after 16 months. An assessment of BA's aphasia was conducted based on the neuropsychological approach and cognitive language models in order to guide selection of speech therapy methods to restore his semantic language skills. Results: An analysis of the assessment results identified a clear advantage of the neuropsychological approach in speech rehabilitation. Unlike the neuropsychological approach, the cognitive language approach to assessment was unable to capture the array of deficits in other higher mental functions caused by specific focal brain lesions or explain the specific impairments of language subsystems. Conclusion: Neuropsychological approaches have greater utility in guiding selection of speech therapy methods that are tailored to different patterns of deficits in language and other higher mental functions.
\end{abstract}

Correspondence: Maria Khrakovskaya; Khrakovm@gmail.com

\section{Developing a paediatric neuropsychological intervention model: Accommodat- ing emerging evidence from neurocognitive, developmental and psychosocial ap- proaches}

\author{
Limond, Jenny ${ }^{1}$; Adlam, Anna ${ }^{2}$ and Cormack, Magnus ${ }^{3}$ \\ ${ }^{1}$ Academic Unit of Mental Health and Wellbeing, Institute of Health and Wellbeing University of Glasgow, \\ Gartnavel Royal Hospital, Glasgow, UK \\ ${ }^{2}$ Department of Psychological Sciences, Norwich Medical School, University of East Anglia, Norwich, UK \\ ${ }^{3}$ CAMHS LD team, NHS Lanarkshire, Motherwell, UK
}

$B$ ackground and aims: Paediatric neuropsychological rehabilitation encounters numerous obstacles both within the clinical and research domains. These obstacles include a limited evidence base due to the heterogeneity of the population, ethical issues of traditional research methods, and paucity of established services. From a clinical perspective, there can be obstacles stemming from family and service resources, as well as individual social, emotional, and behavioural factors. Although models of neuropsychological rehabilitation exist, these are predominantly within the adult domain, and thus neglect the developmental issues that significantly impact on, and interact with, outcomes following paediatric brain injury. The model presented here assimilates literature and evidence from different fields, and provides a systematic approach to deliver and evaluate appropriate care whilst minimising 
the obstacles to successful outcomes. Method: The Medical Research Council guidelines for developing and evaluating complex interventions was used as a framework to guide a review of the intervention literature from child neuropsychology, adult neuropsychology, learning disabilities, education, and mental health. Results: A stepped neurocognitive interventions model was developed from the literature review, taking into account the interactions between established and developing cognitive functions. The model is presented within a holistic framework, incorporating contextual, emotional, and psychosocial issues. Case examples are provided to illustrate how the model can guide clinical practice. Conclusions: The model is evaluated and discussed within the context of delivering clinical interventions to children and young adults with acquired brain injury. Future research directions, based on predictions generated by the model, are also discussed.

Correspondence: Jenny Limond; jennifer.limond@glasgow.ac.uk

\title{
Analysis of intensive inpatient neuro-rehabilitation outcomes using FIM+FAM (UK)
}

Louis, Zacharia ${ }^{1}$ and Florschutz Gerhard ${ }^{2}$

${ }^{1}$ Raphael Medical Centre, neuro rehabilitation of Adults, Hildenborough, UK

${ }^{2}$ The Raphael Medical Centre, Hildenborough, UK

\begin{abstract}
$\boldsymbol{B}$ ackground and aims: This study aimed to analyse the functional outcomes $\mathcal{B}$ of adults with acquired brain injury who attended an intensive inpatient neuro-rehabilitation programme at the Raphael Medical Centre. A retrospective audit was conducted of all admissions over a two-year period (2009-2012) to an inpatient neuro-rehabilitation service utilising an interdisciplinary team approach. Twenty patients who were in receipt of four or more therapies in the intensive inpatient neuro-rehabilitation programme were selected. Method: Routine FIM+FAM (UK) scores were compiled on admission and after 6 months. The FIM+FAM (UK) scale is divided into two broad domains of functioning (motor and cognitive). Descriptive analysis of demographics, patient diagnosis, referral patterns and time since injury to attendance was undertaken. Analysis of FIM+FAM (UK) scores on admission and after 6 months was performed. Results: Complete admission and 6 month follow-up scores were available for all 20 patients. Significant mean improvements in motor (10 point change), cognitive (6.2 point change) and composite FIM+FAM (UK) (16.2 point change) scores were observed, indicating reduced functional dependence following an intensive period of inpatient neuro-rehabilitation. Conclusions: The FIM+FAM (UK) measure demonstrated sensitivity to change across functional domains relevant to goal directed intensive outpatient interdisciplinary neuro-rehabilitation.
\end{abstract}

Correspondence: Zacharia Louis; zachariapl@gmail.com

\section{The Rehabilitation of Dissociative Amnesia}

\author{
Markowitsch, Hans J. ${ }^{1}$ and Staniloiu, Angelica ${ }^{1}$ \\ ${ }^{1}$ Physiological Psychology, University of Bielefeld, P.O.B. 100131, D-33501 Bielefeld, Germany
}

$\boldsymbol{B}$ ackground and aims: Across various cultures dissociative amnesia has been found to occur in response to psychological stress. Not uncommonly dissociative amnesia follows a mild traumatic brain injury. It usually happens without significant brain damage as detected by conventional structural imaging. However, the combined use of functional and newer structural imaging 
has revealed metabolic and micro-structural changes in brain areas involved in mnemonic processing. Dissociative amnesia is commonly retrograde, but anterograde dissociative amnesia may also occur. This paper aims to review the present state of rehabilitation of dissociative amnesia. Method: The review entailed examination of clinical cases and data collected via English, German and French Medline literature searches of empirical studies and original reviews using the keyword combinations of "hysterical", "psychogenic", "functional", "amnesia", "treatment" and "rehabilitation". Results: There are currently no evidence-based treatments for dissociative amnesia, although there have been single case or case series reports of memory improvements after various somatic, psychotherapeutic, or rehabilitative interventions. Several cases of dissociative amnesia were found to follow a chronic course, constituting a major source of disability. Conclusions: Dissociative amnesia can be highly variable with respect to recovery, which may be related to the type and extent of amnesia, form and timeframe of treatment, personal characteristics, therapist variables and external factors (e.g., cultural and socio-economic factors). Combining structural and functional imaging might have potential for the development and evaluation of rehabilitative methods for these conditions.

Correspondence: Hans Markowitsch; hjmarkowitsch@uni-bielefeld.de

\title{
Encephalitis in Childhood and Adolescence: Considerations for Rehabilitation within the Developing Brain
}

\author{
Talbot, Emily ${ }^{1}$ and Starza-Smith, Arleta ${ }^{1}$ \\ ${ }^{7}$ Paediatric Neuropsychology Service, Nottingham University Hospitals NHS Trust, Nottingham, UK
}

$\boldsymbol{B}$ ackground and aims: Encephalitis is a neurological condition characterised by inflammation of brain tissue and has a higher prevalence amongst children. Encephalitis during childhood and adolescence can result in acquired brain injury leading to significant neuropsychological impairment. This has far reaching effects for rehabilitation, recovery and ongoing educational and vocational progress. This paper aims to review the impact of encephalitic brain injury within the developing brain and its recovery trajectory, particularly at key transition points through childhood and adolescence. Method: This paper draws on a group of children and adolescents seen for longitudinal follow-up assessments across the last decade within a specialist paediatric neuropsychology service in a regional acute neurosciences centre in the UK. This cohort of young people includes those who have acquired encephalitic brain injury as an infant and those sustaining the injury in later childhood. Results: Neuropsychological and psychological outcomes for children and families post-encephalitis are dependent on a combination of factors, including: age of onset of encephalitis, duration and severity of illness, age at which assessment is conducted, availability of rehabilitation services, and length of time prior to the initiation of treatment. Conclusions: The support needs of the families of children with an ABI following encephalitis vary according to different stages of the child's development and the demands made on them by the onset of maturity related responsibilities. The need for equitable quality rehabilitation services is emphasised, particularly at key transition points, when Department of Health guidance should be considered.

Correspondence: Emily Talbot; emilytalbot@nhs.net 


\title{
Do people with Acquired Brain Injury ( $\mathrm{ABI}$ ) gain easy access to Neuro Rehabilitation Services across the South East of England?
}

\author{
Tanesh, Bhugobaun ${ }^{1}$ and Drummond, $\mathrm{Di}^{2}$ \\ ${ }^{1}$ Chair KABIF Raphael Medical Centre, Kent, UK \\ ${ }^{2}$ Headway Tunbridge Wells. Kent, UK
}

\begin{abstract}
$B$ ackground and aims: The Kent ABI and Neuro Rehabilitation Strategy Group required information about patients' experiences of accessing acquired brain injury (ABI) services to underpin a comprehensive care pathway strategy from trauma services to long-term neuro rehabilitation care. This study aimed to investigate people's access to neuro rehabilitation services across the South East of England. Method: Questionnaires were sent to 100 patients asking them whether the services were responsive to their needs and if they had easy access to information. A relative or carer involved was asked to complete the questionnaire for those unable to do so. Results: We received 48 responses from those who suffered a brain injury. Of these individuals, $44 \mathrm{had}$ received information about rehabilitation. Of the 48 respondents $61 \%$ received rehabilitation, $35 \%$ had not, and $4 \%$ were unsure. Only $29 \%$ expressed some satisfaction with services after they were discharged. Conclusion: This is the first time such a survey has been undertaken in the South East of England. The results and full analysis will form part of a mapping exercise and service profile across Kent and Medway demonstrating to the Strategy Group and commissioners the need for a clear pathway for inpatients in both the National Health Service and Independent Sectors. It is recommended that this pathway incorporates comprehensive integrated community services for people with $\mathrm{ABI}$ and long-term conditions with neuro rehabilitation needs.
\end{abstract}

Correspondence: Tanesh Bhugobaun; tanesh@raphaelmedicalcentre.co.uk

\section{In Sickness and in Health? The Effect of ABI on Couples' Relationships}

Torjussen, Ingerd ${ }^{1}$

${ }^{7}$ Cognitive Rehabilitation Unit, Brain Injury Department, Sunnaas Rehabilitation Hospital HT, Nesodden, Norway

$B$ ackground and aims: This study aimed to describe the spouse's experience after their partner had been discharged from hospital following acquired brain injury (ABI). The cognitive and psychological difficulties patients experience post-injury are likely to affect the life of their partner. Increased knowledge of spouses' challenges may contribute to better understanding and support from rehabilitation hospitals and the community. Method: A qualitative and descriptive methodological approach was applied. Data were collected through semi-structured interviews. Seven men and women aged 25-60 years were interviewed 4-10 months after hospitalisation. Interviews were taped and transcribed verbatim. The data were analyzed using pragmatic content analysis inspired by Malterud (2006). Results: All spouses reported considerable post-injury changes and emotional stress. Respondents who were able to focus on their present situation seemed to manage everyday life better than those who did not. Lack of communication, intimacy and co-operation were considered frustrating and devastating to their well-being. Spouses reported less time and fewer opportunities to focus on their own needs. The return to home of the patient represented a difficult transition where practical help, psychological support and understanding of their new family situation and their 
surroundings were vital. Conclusion: The findings suggest that partners of patients with ABI require ongoing support in order to adjust and adapt to injury-related challenges.

Correspondence: Ingerd Torjussen; Ingerd.Torjussen@sunnaas.no

\title{
Development of a multidimensional fatigue scale for neurological patients
}

\author{
Visser-Keizer, Annemarie ${ }^{1,2}$; Hogenkamp, Antoinette'; Evers, Marjon² and Schönherr, \\ Marleen ${ }^{1}$ \\ ${ }^{1}$ University Medical Center Groningen, Center for Rehabilitation Beatrixoord, Haren, the Netherlands \\ ${ }^{2}$ University of Groningen, Department of Clinical and Developmental Neuropsychology, Groningen, the \\ Netherlands
}

\begin{abstract}
$B$ ackground and aims: Fatigue is one of the most frequent and disabling complaints in the chronic phase after brain injury. However, nearly all fatigue scales available have either been developed for a non-neurological population or are unidimensional and do not cover the full range of complaints. The aim was to develop a multidimensional scale of fatigue for neurological patients. Method: In-depth interviews were held with 10 stroke patients (months since stroke $\mathrm{M}=49, \mathrm{SD}=41$, range 8-121 months) who presented with fatigue at the rehabilitation center. Three independent reviewers generated items according to the interviews transcribed. Items were presented to patients and professionals. A concept scale containing 57 items was generated, and a priori was divided into the six dimensions - mental fatigue, physical fatigue, fatigue and emotion, time and rest, severity and coping. Results: Data are currently being collected in neurological patients starting outpatient rehabilitation. The first analyses $(n=28)$ showed that the concept scale has good internal reliability (Cronbach's alpha $=.90)$ and significantly correlates with another fatigue scale (CIS20R, $r=.44, p=.03$,) especially with its concentration subscale $(r=.54, p=.005)$. Fatigue was not significantly related to depression or anxiety (HADS depression, $r=.28, p=.19$, HADS anxiety, $r=.35, p=.09$ ). Levels of fatigue did not differ between patient groups (stroke $[n=17]$, traumatic brain injury $[n=5]$ and other brain injury $[n=6])$ and was not significantly related to time since injury $(r=.27, p=.19, M=30, S D=43$, range 2-165 months). Conclusions: The newly developed scale seems a valuable instrument for assessing dimensions of fatigue in neurological patients. It will be further developed based on more data and its psychometric properties.
\end{abstract}

Correspondence: Annemarie Visser; a.visser@cvr.umcg.nl

\section{Integrating the Stuss Frontal Lobe Model into Rehabilitation of Executive Function- ing after Traumatic Brain Injury}

Winegardner, Jill ${ }^{1}$; Ashworth, Fiona ${ }^{1}$; Simblett, Sara ${ }^{2}$; Jennings, Caroline ${ }^{1}$ and Bateman, Andrew ${ }^{1}$

${ }^{1}$ Oliver Zangwill Centre, Ely, UK

${ }^{2}$ Department of Psychiatry, University of Cambridge, Cambridge; National Institute of Health Research (NIHR)

Collaborations for Leadership in Applied Health Research and Care (CLAHRC) for Cambridgeshire and Peterborough

$B$ ackground and Aim: In this paper the application of Stuss's frontal lobe model to the neuropsychological rehabilitation of executive functioning after traumatic brain injury (TBI) is explored. Stuss's $(2007,2011)$ model of 
frontal lobe functioning proposes four domains (executive cognitive, activation regulating, behavioural/emotional self-regulation and meta-cognition) that are linked to neuroanatomical regions and methods of assessments. This paper aimed to extend the model's application to identifying rehabilitation strategies in the TBI population, where non-specific frontal and global neurological consequences are evident. Method: The executive functioning difficulties of five clients with TBI were mapped on to Stuss's domains in order to guide rehabilitation of executive functioning difficulties. Client and carer versions of a modified version of the DEX were used that reflect Stuss's domains along with neuropsychological test performance and staff ratings. Executive functioning interventions were formulated accordingly. Results: The DEX data were converted to logit scores using the four subscales reflecting the domains of Stuss's model and ranked according to perceived level of disability. These were found to be related to performance on neuropsychologist tests relevant to the four domains. Results are considered in terms of conceptualization and intervention for rehabilitation. Conclusions: Stuss's model of frontal lobe functioning is helpful in the conceptualization and rehabilitation of executive functioning difficulties after TBI.

Correspondence: Jill Winegardner; Jill.winegardner@ozc.nhs.uk

\title{
Assessing awareness of deficits after acquired brain injury: a new tool
}

\author{
Winkens, leke ${ }^{1}$; Van Heugten, Caroline ${ }^{1,2}$; Boosman, Hileen ${ }^{3}$ and Visser-Meily, Anne ${ }^{3}$ \\ ${ }^{1}$ Department of Psychiatry and Neuropsychology; Maastricht University; Maastricht; The Netherlands \\ ${ }^{2}$ Department of Neuropsychology and Psychopharmacology; Maastricht University; Maastricht; The Nether- \\ lands \\ ${ }^{3}$ Rudolf Magnus Institute of Neuroscience and Center of Excellence for Rehabilitation Medicine; University \\ Medical Center Utrecht; Utrecht; The Netherlands
}

ackground and aims: Impaired awareness of deficits is an important ob-
stacle to the rehabilitation success of patients with acquired brain injury.
It is associated with less favorable treatment outcomes and influences the types
of treatments therapists can use for alleviating cognitive and behavioral im-
pairments. A reliable assessment of awareness of deficits helps to select those
patients that need interventions to increase awareness of their deficits and can
help therapists to choose appropriate treatment methods. We present a new tool
for the assessment of awareness of deficits after acquired brain injury. Method:
The tool is based on Crosson's hierarchical model of self-awareness and covers
all three levels of awareness of deficits (intellectual, emergent and anticipatory
awareness). It consists of a descriptive classification system with examples
of how to use behavioural observations and discrepancies between patients'
self-reports and their performance on tests. The tool is currently being tested
in a pilot study that includes 30 inpatients from three Dutch rehabilitation cen-
tres. Results: Inter-rater reliability and test-retest reliability will be presented
using simple correlation coefficients and Intra-Class Correlation coefficients.
Convergent validity between results on the Awareness Questionnaire and re-
sults on the new tool will be presented using simple correlation coefficients.
Information regarding the clinical utility of the awareness-instrument will
be presented using qualitative analyses of therapists' opinions. Conclusions:
Therapists have difficulties with assessing awareness of deficit after acquired
brain injury. Data on the clinical utility and the psychometric properties of a
new assessment tool will be presented.

Correspondence: Ieke Winkens; i.winkens@maastrichtuniversity.nl 


\title{
Posture-cognition dual-task performance in patients with traumatic brain injury
}

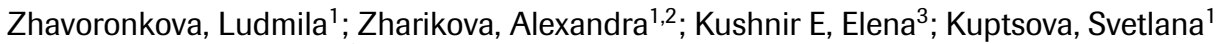
and Mikhalkova, Agdalia ${ }^{3}$

${ }^{1}$ Department of Clinical Neurophysiology, Institute of Higher nervous Activity and Neurophysiology, RAS, Moscow, Russia

${ }^{2}$ Department of neurorehabilitation, Burdenko Neurosurgery Institute, RAMS, Moscow, Russia,

${ }^{3}$ Lomonosov Moscow State University, Moscow, Russia

\begin{abstract}
$B$ ackground and aims: Traumatic brain injury (TBI) is the most common cause of disability in young people. Focal pathology alone cannot explain the functional outcome in these patients. Rather, diffuse axonal injury with functional disconnection is considered to make a major contribution to the neurocognitive and motor deficits resulting from TBI. The aim of this study was to determine changes in postural balance and cognitive perfomance and to understand brain mechanisms underlying dual-task performance in TBI patients. Method: 16 patients $(26.8 \pm 2.8$ years $)$ with long-term TBI and 20 age-matched healthy persons performed cognitive (counting) and postural (on force platform) tasks both separately (single-task) and concurrently (dualtask). The analysis focused on measures of neuropsychological data (executive functions, mental flexibility), stabilographic (velocity and amplitude of centre of pressure) and EEG coherence data for the single and dual-tasks. Results: Compared to controls, TBI patients showed a lower performance of both balance and cognitive performance in single and dual-tasks. The most prominent impairment in TBI patients was evident for the cognitive component on single and dual-tasks. EEG changes such as a diffuse increase in coherence for different spectral bands without specific (normal) regional specificity were observed. However, some of the TBI patients ( 9 persons with minimal clinical impairment) demonstrated a trend for normal EEG reactivity, predominantly for the motor component of the dual-task. Conclusions: This study demonstrated impaired executive functions in patients with long-term TBI. EEG markers of dual-task performance have potential to be used as an approach for monitoring the restoration of cognitive functions in TBI patients during rehabilitation. Supported by RFH 10-06-00114a
\end{abstract}

Correspondence: Ludmila Zhavoronkova; Lzhavoronkova@hotmail.com

\section{SESSION 7: OUTCOME MEASUREMENT}

\section{Comparative Psychometric Analysis of the Mayo-Portland Adaptability Inventory (MPAI-4) in Individuals with Cerebrovascular Accident and Traumatic Brain Injury}

\author{
Malec, James ${ }^{1}$; Kean, Jacob ${ }^{1}$; Altman, Irwin ${ }^{2}$ and Swick, Shannon ${ }^{3}$ \\ ${ }^{1}$ Physical Medicine and Rehabilitation, Indiana University School of Medicine and Rehabilitation Hospital of \\ Indiana, Indianapolis, Indiana USA \\ ${ }^{2}$ Rehab Without Walls ${ }^{\circledR}$, Phoenix, Arizona USA \\ ${ }^{3}$ Rehab Without Walls ${ }^{\circledR}$, Marshall, Michigan USA
}

\begin{abstract}
$B$ ackground and aims: Prior psychometric studies of the Mayo-Portland Adaptability Inventory (MPAI-4) have focused on samples predominantly including individuals with traumatic brain injury (TBI). The aims of this study were: (1) to evaluate the measurement reliability and construct validity of the MPAI-4 in a sample consisting exclusively of patients with cerebrovascular accident (CVA), and (2) to examine differential item functioning (DIF) and differential test functioning (DTF) comparing TBI and CVA samples.
\end{abstract}


Method: MPAI-4 data for 861 individuals post-CVA obtained on admission to community-based rehabilitation were submitted to Rasch analysis. DIF and DTF analyses were performed in comparison to a sample of 603 patients with TBI admitted to the same rehabilitation system. Results: The final calibration in the CVA sample revealed satisfactory Reliability/Separation for Persons (.91/3.16) and Items (1.00/23.64). DIF showed that items for pain, anger, audition, and memory were associated with higher levels of disability for CVA than TBI patients; whereas, self-care, mobility, and use of hands indicated greater overall disability for TBI than for CVA patients. DTF analyses showed a high degree of association between the two sets of items $\left(\mathrm{R}=.92 ; \mathrm{R}^{2}=.85\right)$. Conclusions: The MPAI-4 demonstrates satisfactory psychometric properties for use with individuals with CVA applying for post-acute rehabilitation services. DIF reveals clinically meaningful differences between CVA and TBI groups that should be considered in interpreting results in this population.

Correspondence: James F. Malec, Ph.D., ABPP-Cn, Rp; jmalec@rhin.com

\title{
Measuring support needs across the lifespan after brain injury: the Care and Needs Scales for adults and children
}

\author{
Tate, Robyn ${ }^{1}$; Soo, Cheryl2; Anderson, Vicki ${ }^{2}$; Waugh and Mary-Claire ${ }^{3}$ \\ ${ }^{1}$ Rehabilitation Studies Unit, Sydney Medical School, University of Sydney, Sydney, Australia \\ ${ }^{2}$ Australian Centre for Child Neuropsychology, Murdoch Children's Research Institute, Royal Children's Hos- \\ pital, Melbourne, Australia \\ ${ }^{3}$ Brain Injury Rehabilitation Program, Children's Hospital at Westmead, Sydney, Australia
}

\begin{abstract}
$B$ ackground and aims: Support needs after acquired brain injury vary across multiple dimensions, including type (physical to psychological) and intensity (very little to continual). There are, however, few scales that are designed to directly measure support needs, and consequently the Care and Needs Scale (CANS), along with the paediatric version (PCANS), was developed. Within the taxonomy of the International Classification of Functioning, Disability and Health (ICF), the CANS/PCANS aims to capture the sum total of a person's impairments, activity limitations and participation restriction in terms of Environmental Factors. Method: The CANS (ages 16 years and older) uses a 24-item Needs Checklist that maps to 8/9 ICF Activity/Participation domains to classify intensity of support needs into one of eight hierarchicallyorganised levels. The PCANS applies to ages 5 to 15 years. In order to take account of the developmental spectrum, it uses 105 items which are organised into four forms relating to developmental age bands. Results: Psychometric properties of the CANS have been comprehensively examined. It shows good spread across the eight levels, without significant floor/ceiling effects, has excellent inter-rater $(\mathrm{ICC}=0.93-0.96)$ and test-retest $(\mathrm{ICC}=0.98)$ reliability, and is responsive to change over time, with a large effect size $(\mathrm{ES}=0.98)$. There is evidence of concurrent and discriminant validity with measures of disability. The PCANS was calibrated with normative data from 300 typically developing children and also shows evidence of concurrent and discriminant validity. Conclusions: The CANS/PCANS are psychometrically sound instruments that currently fill a gap as measures of support need after acquired brain injury.
\end{abstract}

Correspondence: Robyn Tate; rtate@med.usyd.edu.au 


\title{
A Structured Interview to Improve the Reliability and Psychometric Integrity of the Disability Rating Scale
}

\author{
Malec, James ${ }^{1}$; Hammond, Flora ${ }^{1,2}$; Giacino, Joseph ${ }^{3}$; Whyte, John ${ }^{4}$ and Wright, Jerry ${ }^{5}$ \\ ${ }^{7}$ Physical Medicine and Rehabilitation, Indiana University School of Medicine and Rehabilitation Hospital of \\ Indiana, Indianapolis, Indiana USA \\ ${ }^{2}$ Carolinas Rehabilitation, Carolinas HealthCare System, Charlotte, North Carolina USA; \\ ${ }^{3}$ Department of Physical Medicine and Rehabilitation, Harvard Medical School and Spaulding Rehabilitation \\ Hospital, Boston, Massachusetts USA \\ ${ }^{4}$ Moss Rehabilitation Research Institute, Philadelphia, Pennsylvania USA \\ ${ }^{5}$ Santa Clara Valley Medical Center, Santa Clara Valley, California USA
}

\begin{abstract}
$B$ ackground and aims: The Disability Rating Scale (DRS) is one of the oldest, most widely used brain injury outcome measures. However, it has: (1) no standard guidelines for administration; (2) substantial skew in postacute samples; and (3) only limited psychometric analyses reported. This study aimed to address these concerns by: (1) developing a standardised interview for telephone administration; (2) assessing the psychometric properties of this interview format (DRS-PI); (3) identifying additional items to reduce skew. Method: DRS-PI questions were developed by consensus of the study investigators, and administered to 406 participants (287 individuals with traumatic brain injury TBI; 119 caregivers) in the U.S. TBI Model System national database during 1-20 year telephone follow-up. Item scores were derived from responses to questions by algorithm. Unnecessary questions were pruned. Results: The DRS-PI correlated highly with the original DRS (intraclass correlation $=.91$ ) and demonstrated satisfactory construct validity and internal consistency (Person Separation/Reliability $=2.51 / .86$; Item Separation/Reliability $=16.72 / 1.00 ;$ Cronbach's alpha $=.83$ ). Both versions showed substantial skew. For original DRS, $42 \%$ of scores were 0 or 1 ; for DRS-PI, 44\%. Adding several items to the DRS-PI including actual employment status further improved its psychometric properties (Person Separation/ Reliability $=3.10 / .91$; Item Separation/Reliability $=21.42 / 1.00$; Cronbach's alpha $=.92$ ) and reduced skew. For the Expanded DRS-PI, 18\% of scores were 0 or 1. Conclusions: The DRS-PI provides an efficient method to assure standardised administration of, and correlates highly with, the original DRS. The addition of several new items, including actual employment status, mitigates skew in postacute samples.
\end{abstract}

Correspondence: James F. Malec, Ph.D., ABPP-Cn, Rp; jmalec@rhin.com

\section{International consensus on outcome measures for neuropsychological rehabilita- tion research in acquired brain injury: preliminary results}

van Heugten, Caroline ${ }^{1}$

${ }^{1}$ School for Mental Health and Neuroscience, and Department of Neuropsychology and Psychopharmacology, Maastricht University, the Netherlands

$\boldsymbol{R}$ ackground: During the last meeting of the Special Interest Group on Neuropsychological Rehabilitation (July 2011), the lack of consensus about which outcome measure to use to evaluate the effectiveness of neuropsychological interventions was taken as a starting point for a joint project. Objective: To reach international consensus on outcome measures for neuropsychological rehabilitation research in acquired brain injury. Methods: Members of the SIG-NR were invited to participate in a study group using an iterative, collaborative, evidence-based approach to identify the best of currently available 
outcome measures. The first steps were taken during the SIG-NR meeting in July 2011 and included formation of the study group and formulating a global project plan. The next steps involved: 1) formulating a concrete project plan, 2) definition of the scope of the project, 3) a first Deplhi-round to determine the domains of outcome, and 4) a first Delphi-round to establish the set of characteristics of a good quality outcome measure. Discussion and conclusions: The results of the next steps in the joint project will be presented at the SIG-NR meeting in 2012 and will eventually be published in an international well-accepted journal in the field of neuropsychological rehabilitation as recommendations for future research.

Correspondence: Caroline van Heugten; c.vanheugten@ maastrichtuniversity.nl

\title{
SESSION 8: DATABLITZ - PSYCHOLOGICAL AND BEHAVIOURAL FUNCTIONING
}

\section{Do in-patients with acquired brain injury (ABI) and complex needs show less awareness of their difficulties than community dwelling patients with $A B I$ ?}

\author{
Wilson Barbara A. ${ }^{1}$; Florschutz, Gerhard ${ }^{2}$ and Bateman, Andrew ${ }^{1}$ \\ ${ }^{1}$ The Oliver Zangwill Centre, Princess of Wales Hospital, Ely, UK \\ ${ }^{2}$ The Raphael Medical Centre, Hildenborough, UK
}

\begin{abstract}
$B$ ackground and aims: The European Brain Injury Questionnaire (EBIQ) and the Dysexecutive Questionnaire (DEX) have good psychometric properties (Simblett \& Bateman, 2011) and can be used to determine patients' perceptions of their problems. It is also possible to look at discrepancies between self-rating of difficulties and ratings from an independent person who knows the patient well, to estimate level of insight. We wanted to know whether patients with complex needs (PCN) had less insight and showed a greater discrepancy between self and independent ratings than patients who were attending a community rehabilitation service (CRS). Method: The EBIQ and the DEX were administered to a large group of patients $(>250)$ from a CRS and to a small group $(\mathrm{N}=23)$ of $\mathrm{PCN}$ residing in an in-patient unit. A relative or carer who knew the patient well also completed the questionnaires. Results: On both measures the PCN rated themselves as having fewer problems than the CRS patients. The independent ratings were not significantly different for the two groups. The PCN were significantly discrepant from carers for both measures and more discrepancy was observed between self and independent ratings than found for the CRS patients. Conclusions: Despite having more physical and cognitive problems than the CRS patients, PCN rated themselves as having fewer difficulties and showed a greater discrepancy between their own scores and the independent ratings. We have shown that these measures can be used with the two populations to determine patients' perceptions of their difficulties and as a rough estimate of level of insight.
\end{abstract}

Correspondence: Barbara A. Wilson; barbara.wilson00@gmail.com 


\title{
Awareness of deficits and treatment motivation after acquired brain injury
}

Smeets, Sanne M.J. ${ }^{1}$; Wolters Gregório, Gisela ${ }^{1}$; Ponds, Rudolf W.H.M. ${ }^{1,2}$ and van Heugten, Caroline M. ${ }^{1,3}$

${ }^{1}$ Department of Psychiatry and Neuropsychology, Maastricht University, Maastricht, The Netherlands

${ }^{2}$ Department of Psychiatry and Psychology, Maastricht University Medical Center (MUMC) / Alzheimer Center Limburg, Maastricht, The Netherlands

${ }^{3}$ Department of Neuropsychology and Psychopharmacology, Faculty of Psychology and Neuroscience, Maastricht University, Maastricht, The Netherlands

\begin{abstract}
$B$ ackground and aims We investigated the level of awareness of deficits and treatment motivation in a group of patients with acquired brain injury (ABI) who were referred to outpatient mental health clinics because of behavioral and/or emotional problems. It is important to investigate awareness and treatment motivation because they are factors that might influence treatment outcome. Method To evaluate awareness we used the discrepancy in answers between patients (P) and significant others (SOs) and/or clinicians (C) on the Awareness Questionnaire (AQ), Patient Competency Rating Scale (PCRS) and a clinical judgment scale $(1=$ completely impaired to $5=$ not at all impaired). Treatment motivation was measured with the Motivation for Traumatic Brain Injury Rehabilitation Questionnaire (MOT-Q; scale -62 to 62) and a Visual Analogue Scale (VAS; scale 1 to 10). Results Preliminary analysis included 89 patients $(68.5 \%$ male), mean age $44.6(S D=13)$. Mean time since injury was 10.5 years $(S D=10.4)$. Most patients had no or mild impaired awareness of deficit $(M(S D)$; AQ P-SO = $2.0(8.1)$; AQ P-C $=1.1(0.3)$; PCRS P-SO $=2.7$ (16.4); and PCRS P-C $=1.1(0.2)$. Patients had high treatment motivation: mean VAS $8.1(S D=2.2)$ and MOT-Q $16.6(S D=14.7)$. Depending on the measurements used awareness and treatment motivation correlated moderately to strongly $(r=-.30-.60)$, meaning that patients with better awareness were more motivated. Conclusions Surprisingly, these preliminary results indicate that this sample of ABI patients had good awareness of their deficits. This correlated with higher treatment motivation. Further interpretations of the results will be discussed.
\end{abstract}

Correspondence: Sanne Smeets; smj.smeets@ maastrichtuniversity.nl

\section{Passive coping is maladaptive in patients with psychiatric and behavioural prob- lems due to acquired brain injury}

\author{
Wolters Gregório, Gisela ${ }^{1}$; Smeets, Sanne M.J. ; Ponds, Rudolf W.H.M. ${ }^{1,2}$ and van Heugten, \\ Caroline M. ${ }^{1,3}$ \\ ${ }^{1}$ Department of Psychiatry and Neuropsychology, Maastricht University, Maastricht, The Netherlands \\ ${ }^{2}$ Department of Psychiatry and Psychology, Maastricht University Medical Center (MUMC) / Alzheimer Cen- \\ ter Limburg, Maastricht, The Netherlands \\ ${ }^{3}$ Department of Neuropsychology and Psychopharmacology, Maastricht University, Maastricht, The Nether- \\ lands
}

\begin{abstract}
$B$ ackground and aims: Coping has shown promise in influencing psychosocial functioning in patients who mainly suffer from cognitive difficulties due to brain injury. However, studies have yet to investigate relationships between coping and psychosocial functioning in patients whose behavioural and/or psychiatric difficulties are most prominent, nor in their caregivers. It is important to investigate these relationships in order to develop effective treatment programmes for this population. Method: Patients in the chronic phase ( $>6$ months after injury) and their caregivers were recruited from consecutive
\end{abstract}


admissions to outpatient units of 4 mental health centres in The Netherlands. Patients completed the Utrecht Coping List, Patient Health Questionnaire, and the Life Satisfaction Questionnaire. Also, the caregivers filled out the Utrecht Coping List and Life Satisfaction Questionnaire, and the Caregiver Strain Index. Data were analysed with linear regression. Results: In total 89 patients and 59 caregivers participated. Patients who used more passive coping styles reported a lower quality of life and more depressive complaints $(p<0.001)$. Caregivers who used more passive coping styles reported lower quality of life $(\mathrm{p}<0.01)$ and more strain $(\mathrm{p}<0.001)$. There were no effects of coping of the caregiver on psychosocial functioning of the patient $(\mathrm{p}>0.05)$. Conclusions: Passive coping was shown to be maladaptive in patients with prominently psychiatric and behavioural problems after brain injury. Psychosocial functioning could be improved by measuring coping on admission to mental health centres, and by changing passive styles into more productive styles.

Correspondence: Gisela Wolters Gregório; g.woltersgregorio@maastrichtuniversity.nl

\title{
Alcohol use one to three years following traumatic brain injury
}

Spitz, Gershon ${ }^{1}$ and Ponsford, Jennie ${ }^{1}$

${ }^{1}$ School of Psychology and Psychiatry, Monash University, 3800, Australia

\begin{abstract}
$B$ ackground and Aims: Alcohol use is implicated in a significant proportion of traumatic brain injuries (TBI). Between seven and 26\% of individuals continue heavy use of alcohol 1-5 years post-injury (Parry-Jones et al., 2006). The current study examined alcohol use between one and three years following TBI and the influence of premorbid alcohol use, injury severity, and demographic variables. Method: 132 individuals with mild to severe TBI completed the Alcohol Use Disorders Identification Test (AUDIT) during inpatient rehabilitation - as a measure of pre-injury alcohol use-and at one, two, and three years post-injury. 132 healthy controls were also assessed on one occasion. There were no significant differences in age, gender or education between the groups. Multilevel modelling was used to model the trajectory of alcohol use over time. Results: No difference was found between TBI and control participants in regards to pre-injury alcohol use, though TBI participants displayed significantly reduced alcohol consumption at one, two, and three years post-injury. Nevertheless, a significant increase was apparent between one and three years following TBI, with fewer days of post-traumatic amnesia and higher pre-injury alcohol use predicting greater alcohol consumption. Interestingly, a significant interaction indicated that whereas TBI participants drinking at hazardous levels pre-injury displayed a decline in consumption after injury, those individuals that displayed normal alcohol use pre-injury showed increased consumption between one and three years post-injury. Discussion and Conclusion: These findings suggest that overall, individuals with TBI reduce their alcohol consumption, in line with advice provided by their rehabilitation physicians. However, individuals with milder injuries and those drinking at less than hazardous levels may require greater focus as they are most at risk of increased post-injury drinking.
\end{abstract}

Correspondence: Gershon Spitz; Gershon.Spitz@monash.edu 


\title{
Neuropsychological rehabilitation in intellectual disability: strengths, weaknesses and theoretical contribution to treatment definition in complex rehabilitation inter- ventions
}

\author{
Sgaramella, Teresa Maria ${ }^{1}$; Ruggero, Anna ${ }^{1}$; Santise, llaria ${ }^{2}$ and Barone, Caterina ${ }^{3}$ \\ ${ }^{1}$ Dipartimento di Psicologia dello Sviluppo e della Socializzazione, Università di Padova, Italy \\ ${ }^{2}$ Centro di Ateneo Ricerca e Servizi, Disabilità, Trattamento e Integrazione, Padova, Italy \\ ${ }^{3}$ Cooperativa Sociale Magnolia-Onlus, Piove di Sacco, Padova, Italy
}

\begin{abstract}
$B$ ackground and aims: This paper analyses the relevance and contribution of a theory based neuropsychological rehabilitation in intellectual disability. Specifically, it describes and evaluates a programme which was aimed at improving abilities involved in money knowledge and use. Method: The program was developed in three steps: cognitive rehabilitation (graduated exercises divided into sub-programs, each corresponding to a sub-component, i.e. basic number and money knowledge) which enabled participants to acquire basic knowledge; strategy learning (simple calculation and money use); and ecological rehabilitation, including analyses of scenarios and simulations of real-life situations, which aimed to transfer the knowledge and strategies learned to everyday life. Eleven young adults with mild-to-moderate intellectual disability of different aetiology were included in two weekly sessions of a five month rehabilitation program. Baseline assessments were used to measure the effectiveness of the rehabilitation, with outcome measures including direct learning of practiced material, improved money knowledge and use, and a generalization to related cognitive processes. Results: Although the outcomes varied across participants, the program was found to be effective in producing significant "changes" in money knowledge and use (naming, estimate and ordering). Participants also showed generalization of skills to everyday life as evidenced by skills shown in the activity of shopping. These effects were found in participants with mild intellectual disability and were maintained at three months follow-up. Conclusions: An analysis of active ingredients and principles used to overcome learning difficulties, together with behavioral operations, procedures and materials used, provides greater understanding of key elements for a successful neuropsychological rehabilitation program. The results also support the usefulness of neuropsychological rehabilitation in intellectual disability.
\end{abstract}

Correspondence: Teresa Maria Sgaramella; teresamaria.sgaramella@unipd.it

\section{SESSION 9: ADVANCES IN MEASURING SOCIAL AND EMOTIONAL WELL-BEING}

\section{Improving measurement of coping style following acquired brain injury}

\footnotetext{
Simblett, Sara. K. ${ }^{1,2}$; Gracey, Fergus ${ }^{1,3}$; Ring, Howard ${ }^{1,2}$ and Bateman, Andrew ${ }^{1,3}$

${ }^{7}$ National Institute for Health Research (NIHR) Collaborations for Leadership in Applied Health Research and Care (CLAHRC) for Cambridgeshire and Peterborough, Cambridgeshire and Peterborough NHS Foundation Trust, Cambridge, UK.

${ }^{2}$ Department of Psychiatry, University of Cambridge, Cambridge, UK.

${ }^{3}$ Oliver Zangwill Centre for Neuropsychological Rehabilitation, Cambridgeshire Community Services NHS Trust, Ely, UK.
}

$B$ ackground and aims: The effects of coping style on emotional adjustment following acquired brain injury (ABI) are well researched. Avoidant and emotion-focused coping are associated with poor outcomes, and task-oriented coping with better outcomes. Interventions aimed at improving coping are 
gaining increased attention. To accurately assess the effectiveness of such interventions, measurement precision is paramount. Methods: Psychometric properties of the Coping Inventory for Stressful Situations (Endler \& Parker, 1990) were investigated using Rasch analysis of responses from a sample of adults with ABI $(n=201)$. The internal consistency reliability and construct validity, including the dimensional structure, of the scale was examined. $R e$ sults: All originally proposed subscales were found to deviate significantly from the response pattern expected by the Rasch model, and therefore, did not display valid and reliable interval-level measurement properties (Task: $X^{2}=100.6, p<.001$; Emotion: $X^{2}=116.8, p<.001$; Avoidance: $X^{2}=66.4$, $p<.001$; Social diversion: $X^{2}=20.4, p<.05$; Distraction: $X^{2}=44.8, p$ $<.001)$. The sample failed to distinguish between five response categories for $34(71 \%)$ of the items. Three valid and reliable subscales were derived: task-oriented $\left(X^{2}=29.7, p=.13\right)$, emotion-oriented $\left(X^{2}=34.2, p=.13\right)$ and avoidance-oriented $\left(X^{2}=13.8, p=.54\right)$. Conclusion: Coping style is important to emotional adjustment following $\mathrm{ABI}$ and is likely to be a focus of future research and clinical intervention. Three distinct coping domains were identified. This analysis will contribute to better understanding and measurement of coping style following ABI.

Correspondence: Sarah Simblett; ss879@medschl.cam.ac.uk

\title{
Measuring communication-specific coping: Development and evaluation of the Communication-Specific Coping Scale
}

\author{
Douglas, Jacinta M. ${ }^{1}$ and Mitchell, Carren ${ }^{1}$ \\ ${ }^{7}$ Human Communication Sciences, La Trobe University, Victoria, Australia
}

\begin{abstract}
$\boldsymbol{R}$ ackground and aims: Self and close other reports of ability provide a 0 valuable and time-efficient means of assessment during rehabilitation. The Communication-Specific Coping Scale (CommSpeCS) is a new scale designed to measure the productive and non-productive coping strategies people use in situations characterised by communication breakdown. Productive communication-specific strategies enhance message transfer and facilitate social participation; non-productive strategies do little to resolve problems and frequently result in negative social interaction. This research aimed to psychometrically evaluate the CommSpeCS. Method: Evaluation of the CommSpeCS has involved two studies. First, internal consistency and temporal stability were evaluated on a normative control sample of 118 healthy adults and close others. Second, the ability of the CommSpeCS to distinguish between a group of 26 adults with traumatic brain injury (TBI) and a matched control group of 26 neurologically-normal adults was then evaluated. Results: Internal consistency and test-retest reliability coefficients exceeded 0.80 in the normative sample for self and close other versions of the scale. Results of the clinical comparison also supported the clinical utility of the CommSpeCS. The scale was able to distinguish between the TBI group and the control group. Further, significant differences between the groups emerged from both the perspectives of the injured adults and the perspectives of their close others. Conclusions: Results support the use of the CommSpeCS as a reliable measure of communication-specific coping. Accurate measurement of communicationspecific coping strategies enables rehabilitation efforts to be directed towards increased use of productive strategies and decreased use of non-productive strategies in the context of communication breakdown.
\end{abstract}


Correspondence: Jacinta Douglas; J.Douglas@latrobe.edu.au

\title{
Exploring the nature of self-esteem after Acquired Brain Injury
}

Longworth, Catherine, E. ${ }^{1}$; Deakins, Joseph ${ }^{2}$; Rose, D. ${ }^{3}$ and Gracey, Fergus 1,4 $^{1,4}$

${ }^{1}$ Oliver Zangwill Centre for Neuropsychological Rehabilitation, Cambridgeshire Community Services (NHS) Trust, Ely Cambridgeshire, UK

${ }^{2}$ Department of Psychology, Royal Holloway, University of London, London, UK

${ }^{3}$ Department of Psychology, University of Surrey, Guildford, UK

${ }^{4} \mathrm{NIHR}$-Collaborations for Leadership in Applied Health Research and Care for Cambridgeshire and Peterborough (CLAHRC-CP), Cambridge University Dept of Psychiatry, Cambridge, UK

\begin{abstract}
$B$ ackground and aims: Acquired Brain Injury (ABI) often results in negative changes in self-concept and increased rates of low self-esteem, depression or anxiety. Previous ABI research has used unidimensional selfesteem measures despite evidence that self-esteem differs across domains of self-concept in the non-brain injured population. This study aimed to validate an established multidimensional measure of self-esteem in people with ABI, to assess whether self-esteem is multidimensional after ABI and, if so, to identify and describe the dimensions. Method: A sample of adults with ABI referred for assessment for comprehensive intensive rehabilitation $(\mathrm{N}=80)$ completed the Robson Self-Concept Questionnaire (RSCQ) and the Hospital Anxiety and Depression Scale. Correlation and factor analyses were conducted to address study aims. Results: $28 \%$ of the sample had low self-esteem and this was associated with severity of ABI (lower self-esteem in mild-to-moderate ABI than in severe $\mathrm{ABI}$ ), depression and anxiety. Internal consistency of the RSCQ was good. Factor analysis found four dimensions of self-esteem after ABI labelled 'perceived self-worth', 'likeability', 'resilience' and 'confidence'. This result differed from the factor solution identified in non-ABI analysis of the measure. Conclusions: The results suggest that the RSCQ is a reliable measure of self-esteem after ABI enabling evaluation of multiple dimensions of self-esteem. A multidimensional view of self-esteem after ABI was supported although dimensions differed from those previously identified. Consistent with an established cognitive behavioural model, self-esteem was associated with depression and anxiety, warranting further research on interventions to improve self-esteem as a means of improving emotional outcomes post ABI.
\end{abstract}

Correspondence: Catherine Longworth; Catherine.Ford@ozc.nhs.uk

\section{Wearable biofeedback technology in emotional work within neuro-rehabilitation}

O'Neill, Brian $^{1,2}$; Findlay, Gemma ${ }^{1}$ and Goodfellow, Rebecca ${ }^{1}$

${ }^{1}$ Brain Injury Rehabilitation Trust, Glasgow, UK

${ }^{2}$ University of Stirling, Stirling UK

$\boldsymbol{B}$ ackground and aims: Assistive technology for cognition (ATC) is re$B$ viewed with specific reference to technologies useable in emotional work after brain injury. A recent systematic review utilising the International Classification of Functioning highlighted a relative lacuna of evidenced technologies supporting emotional functions. Emotional dysregulation is a key variable in understanding challenging sequelae of brain injury including irritability, verbal and physical aggression, self-injury, impulsivity, poor life decisions and relationship difficulties. Recent ATC developments include wearable systems that provide biofeedback to the user on heart-rate variability, a marker for 
autonomic nervous system dynamics. Method: Three $\mathrm{n}=1$ case studies investigated the effectiveness of a biofeedback device measuring heart rate variability and coherence (EmWave2) in an ABA design. In an in-patient rehabilitation setting, three individuals with challenging behaviour attributable to severe brain injury and associated with high arousal used the EmWave 2 device for 10-20minutes per day in the intervention phase. Outcome variables were incidence of verbal and physical aggression, self-injurious behaviour and use of per-required-need benzodiazepines. Results: In each case there was a reduction in the target behaviours attributable to the intervention. Return to baseline suggests lasting effects. Randomisation statistics are reported. Conclusions: The problems of emotional dysregulation challenge the individual and others. Interventions to date are typically pharmacological, psychological or social restriction. Assistive technology for cognition offers a fourth treatment modality where individuals can become more aware of and lower their arousal levels with lasting effects on target behaviours.

Correspondence: Brian O’Neill; brian.oneill@thedtgroup.org

\section{SESSION 10a: DATABLITZ - BRAIN INJURY OUTCOMES IN CHILDHOOD AND ADO- LESCENCE}

\section{Encephalitis-Related Sleep Difficulties in Childhood - Future Research and Clinical Implications}

Talbot, Emily ${ }^{1}$; Starza-Smith, Arleta ${ }^{1}$ and Hart, Aidan ${ }^{2}$

${ }^{1}$ Paediatric Neuropsychology Service, Nottingham University Hospitals NHS Trust, Nottingham, UK

${ }^{2}$ Trent Doctorate in Clinical Psychology, University of Lincoln, Lincoln, UK

$B$ ackground and aims: Sleep difficulties are commonly reported in children post-encephalitis. Following our 2010 study of health-related quality of life (HRQL) of children post-encephalitis, further exploration of sleep difficulties in the context of HRQL emerged as an area of priority through a review of the literature within this topic area. Further specific research to consider rehabilitation needs of children/adolescents and their families are discussed. Method: We review findings of our study exploring parent/carer reported HRQL for children/adolescents post-encephalitis. Participants were recruited through the Encephalitis Society with 33 parents/carers of children/adolescents, aged $8-15$ years, completing the Pediatric Quality of Life Inventory ${ }^{\mathrm{TM}}$ (PedsQL ${ }^{\mathrm{TM}}$ ), proxy measures of memory and executive function, as well as demographic/illness specific questions including asking whether their child experienced sleep difficulties. An analysis of the parent responses to this question was conducted within the framework of a literature review of sleep difficulties post-childhood encephalitis. Results: $64 \%$ of parents/carers reported sleep difficulties for their child post-encephalitis. Sleep difficulties were found to significantly correlate with parent/carer reported HRQL. There is a dearth of studies exploring sleep problems post-childhood encephalitis. Of the literature available the impact of sleep difficulties on the child/adolescent and family well-being are highlighted. Conclusions: Sleep difficulties are frequently reported following childhood encephalitis and relate significantly to parent/carer reported HRQL which impacts on rehabilitation. We propose further research to explore this topic area, including investigation of sleep disorders, current treatments and the impact of sleep difficulties on 
children/adolescents and their families. We highlight implications for clinical practice and rehabilitation.

Correspondence: Emily Talbot; emilytalbot@nhs.net

\title{
Cooperative learning and metacognition (Metacognitive Dimension) in pre- adolescents with acquired brain injury (ABI): Improving self-concept, self- regulation and quality of life
}

\author{
Braga, Lucia Willadino ${ }^{1}$ \\ ${ }^{1}$ SARAH Network of Neurorehabilitation Hospitals, Brasilia, Brazil
}

\begin{abstract}
$B$ ackground and aims: The aim of this randomized clinical trial study was to evaluate the efficacy of an intervention program based on social mediation, cooperative learning and metacognition (Metacognitive Dimension) in pre-adolescents with acquired brain injury (ABI). Methods: Participants were pre-adolescents with $\mathrm{ABI}$ divided into two groups: experimental and control. Evaluations were conducted 3 months after the start of the intervention, using the Evaluation Scale of Elementary School Learning Strategies (ESESLS) to assess metacognitive strategies, Self-Concept Scale for Children (SCSC) and Behavioral Rating Inventory of Executive Functions (BRIEF). Results: Overall, the experimental group had superior outcomes to the control. The results for metacognitive strategies (ESESLS) and self-concept (SCSC) were better in the experimental group than in the control group $(\mathrm{p}<0.05)$. No significant differences were revealed between the groups on the BRIEF, although we observed better results in the experimental group for 5 sub items of the scale, in the broader index and the global executive composite score. Conclusion: Three months of intervention based on cooperative learning supported preadolescents with acquired brain injury to develop metacognitive strategies and improve their self-concept, thereby helping to empower them in their social relationships.
\end{abstract}

Correspondence: Lucia Willadino Braga; luciabraga@sarah.br

\section{The effect of age at injury and socio-economic status on recovery after childhood severe traumatic brain injury: results of a prospective study}

\author{
Chevignard, Mathilde ${ }^{1,2}$; De Agostini, Maria ${ }^{3}$; Escolano Sylvie ${ }^{3}$; Laurent-Vannier Anne ${ }^{1}$; \\ Lancien Sabine $^{1}$ and Meyer Philippe ${ }^{4}$ \\ ${ }^{7}$ Rehabilitation Department for Children with Acquired Brain Injury, Hôpitaux de Saint Maurice, Saint Maurice, \\ France \\ ${ }^{2} E R-6$, Pierre et Marie Curie University, Paris, France \\ ${ }^{3}$ CESP U 1018 Inserm, Villejuif, France \\ ${ }^{4}$ Paediatric Anesthesiology Department, Hôpital Necker Enfants Malades, 75007 Paris, France
}

$B$ ackground and aims: The purpose of this study was to evaluate cognitive and functional outcomes of children who sustained severe TBI over 24 months ago and to determine factors predicting outcomes. Method: Sixty-five children were included in a prospective longitudinal cohort. Assessment was conducted at 3 and 24 months, and included age appropriate Wechsler Intelligence Scales and the Pediatric Injury Functional Outcome Scale (PIFOS). Socio-economic status (SES) was assessed by parents' education. Results: Children (mean age at injury 8.12 years $[S D=4.6]$ ) were divided into two age groups ( $<6$ years, $n=23$ and 6 years, $n=42$ ). At 3 months post-injury, mean 
Full-Scale IQ (FSIQ) fell one standard deviation below the norms $(M=85.0$; $S D=16.0$ ) in both age groups. All children had difficulties on the PIFOS. At 24 months, IQ had significantly improved in the older age group $(M=92.9$; $\mathrm{SD}=21.0)$, but not in the younger group $(M=83.15 ; \mathrm{SD}=21.0)$. At both assessment times, IQ was highly dependent on SES $(\mathrm{p}<0.0001)$. PIFOS scores improved at 24 months; however, $90 \%$ of the older age group still displayed difficulties, versus $100 \%$ of the younger age group. Change over time was predicted by age at injury for IQ $(\mathrm{p}=0.03)$ and by injury severity for the PIFOS $(\mathrm{p}<0.01)$. Conclusion: After severe TBI, improvement was found at 24 months, but only in the older children. Cognitive and functional outcomes were related to parental education on all assessments. However, unlike in other studies, this effect was stable over time, although parental education did not significantly moderate improvement over time.

Correspondence: Mathilde Chevignard; m.chevignard@hopitaux-stmaurice.fr

\section{Long-term outcomes following childhood and adolescence acquired brain injury: A characterisation study of adult survivors}

Béasse, Solenn ${ }^{1}$; Noublanche, Nicole ${ }^{2}$; Lambert, Ariéle ${ }^{2}$; De Reuck, Fanny ${ }^{3}$; Katara, Ravi ${ }^{3}$; Croisiaux, Christine ${ }^{3}$ and Chevignard Mathilde ${ }^{4,5}$

${ }^{1}$ Foyer d'Accueil Médicalisé La Maison du Sophora, Gauchy, France

${ }^{2}$ Brain injury mutualist pole Arceau Anjou, Angers, France

${ }^{3}$ La BRAISE, structures dedicated to long-term care and support of brain-injured adults, Bruxelles, Belgium

${ }^{4}$ Rehabilitation Department for Children with Acquired Brain Injury, Saint Maurice Hospital, Saint Maurice, France

${ }^{5}$ ER - 6; Pierre et Marie Curie University, Paris, France

ackground and aims: Acquired brain injury (ABI) sustained during child-
hood or adolescence is known to impact on long-term community inte-
gration and participation. The purpose of this study was to characterise this
population more precisely, and to consider how support needs may vary ac-
cording to age at injury. Methods: Aetiology of ABI and age at injury were
collected for 1023 adults (aged 18 - 60 years) registered in 12 French and Bel-
gian medico-social structures or services for adults with ABI in 2009 . Sixty-six
professionals working within these institutions answered a questionnaire about
the similarities and differences that they observed in the clients according to
age at injury. Results: Overall, $28 \%$ of the patients had sustained an ABI
between birth and 20 years of age. The highest proportion of this subgroup
was found in institutions providing sheltered work and/or accommodation.
Results of the questionnaires indicated that injury in childhood was associated
with cognitive, behavioural and emotional difficulties (e.g., immaturity), and
lower levels of autonomy for basic and instrumental activities of daily living.
These individuals were also reported to experience more difficulty (relative
to those with adult-onset ABI) in participating in projects related to future
life. Conclusion: The proportion of adults with injuries sustained in childhood
was surprisingly high. Moreover, the presentation of those with paediatric
ABI differed from those who sustained ABI in adulthood. It is recommended,
therefore, that cognitive, emotional, and behavioural development, as well as
development of autonomy, is considered when organising long-term care for
survivors of childhood ABI. 
This work was performed following an initiative of the European Brain Injury Society (EBIS).

Correspondence: Solenn Béasse; sbeasse.psy@gmail.com

\title{
SESSION 10b: DATABLITZ - PSYCHOLOGICAL AND SOCIAL FUNCTIONING
}

\section{Soothing the injured brain with compassion: Using the compassionate mind ap- proach in holistic neuropsychological rehabilitation}

\author{
Ashworth, Fiona ${ }^{1}$; Longworth Catherine, $\mathrm{E}^{1}$ and Clarke Alexis ${ }^{1}$ \\ ${ }^{1}$ The Oliver Zangwill Centre for Neuropsychological Rehabilitation, Cambridgeshire Community Services NHS \\ Trust, Ely, UK
}

\begin{abstract}
$B$ ackground and Aims: Psychological difficulties after an acquired brain injury (ABI) are common (Kim et al., 2007). Clinical diagnoses of psychological disorders often do not encompass the complex cognitive, emotional, and psychosocial consequences experienced by individuals with brain injury. Gilbert's compassionate mind approach, which focuses on transdiagnostic processes such as self-criticism, is emerging as a useful model for conceptualising and guiding intervention approaches for those with emotional difficulties after ABI (Ashworth, Gracey \& Gilbert, 2011). This framework has been used within our holistic neuropsychological rehabilitation programme to provide a psycho-educational group on emotional difficulties after ABI and individual psychotherapy. The primary objective of this paper is to present initial outcome data from these interventions. Method: Questionnaire data (Forms of Self-Criticism/Attacking and Self-Reassurance Scale (FSCRS) from 12 individuals with ABI who received both group and individual psychotherapy sessions using the compassionate mind approach are examined. These results are supplemented by a qualitative analysis of interviews regarding individuals' experience of the psycho-education group. Results: The findings suggest that individuals high in self-criticism report a decrease in self-criticism and an increase in the ability to self-reassure at the end of the rehabilitation program. We consider the impact of this intervention on psychological wellbeing more generally, alongside their experiences of the psycho-educational group. Conclusions: These initial data provide preliminary support that focusing psychological interventions on underlying transdiagnostic processes after ABI can be effective. These clinical findings support the utility of the compassionate mind model for conceptualising and guiding interventions for emotional difficulties after ABI.
\end{abstract}

Correspondence: Fiona Ashworth; Fiona.Ashworth@ozc.nhs.uk

\section{Changes in community travel patterns after acquired brain injury and its impact on quality of life}

\author{
Nice, Laura ${ }^{1}$ and Powell, Theresa ${ }^{1,2}$ \\ ${ }^{1}$ School of Psychology, University of Birmingham, UK \\ ${ }^{2}$ Birmingham Community Healthcare Trust, Birmingham, UK
}

$B$ ackground and aims: Levels of independence and community integration are important for life satisfaction after brain injury (Johnston et al., 2005) but community integration usually relies on the ability to travel outside the home. Anxiety may also play a role in community travel patterns (Sohlberg et 
al, 2005). The study aims were to examine changes in community travel after $\mathrm{ABI}$ and investigate the role of anxiety in these changes (controlling for level of disability/independence) as well as determine the impact on quality of life. Method: 56 participants with ABI (mean time post injury 12 years) completed three questionnaires: a community travel questionnaire; Nottingham Extended Activities of Daily Living Index; and the Ferrans and Powers Quality of Life Index. Results: $76 \%$ of participants reported a change in travel patterns after injury (69\% less travel, $7 \%$ more travel, $24 \%$ no difference in travel). The overall frequency of trips into the community reduced significantly, especially the number of journeys made unaccompanied, although the number of accompanied journeys reduced to less extent. The decrease in unaccompanied journeys correlated with level of anxiety and independence, although anxiety was the main predictor. The amount of travel in the community (alone and accompanied) post injury was a better predictor of quality of life than independence, although the amount of variance explained was small. Conclusions: Travel patterns changed significantly after injury with fewer journeys occurring overall and proportionally more accompanied journeys. Anxiety was found to be an important barrier to people going out alone. Given the associations with quality of life, strategies to enhance community travel require greater attention in rehabilitation.

Correspondence: Laura Nice; 1xn964@bham.ac.uk

\title{
An exploration of the experience of self in the social world for men following traumatic brain injury (TBI)
}

\author{
Freeman, Anita ; Adams, Malcolm'; Adlam, Anna ${ }^{1}$ and Ashworth, Fiona ${ }^{2}$ \\ ${ }^{1}$ University of East Anglia, Norwich, UK \\ ${ }^{2}$ The Oliver Zangwill Centre, Prince of Wales Hospital, Cambridgeshire, UK
}

\begin{abstract}
$B$ ackground and aims: The lived experience of individuals with TBI has been difficult to fully capture through quantitative enquiry. The aim of this study was to encourage individuals to talk freely of their experience of changes in self in relation to their inner and social world, and to provide a depth of description of their lived experiences following TBI. Method: Semistructured interviews were conducted with nine males with TBI (aged 22 to 59 years) recruited through a neuropsychological rehabilitation centre. Transcriptions of their accounts were analysed using thematic analysis, identifying dominant themes related to their described emotional experience of self in the social world. Results: The findings revealed themes of 'abnormality', 'hidden', 'old-me, new me' and 'others treat me differently', which were related to the men's experience of self following TBI, and themes of responses to experiences as 'self-criticism', 'need to be as others want me to be' and 'withdrawal'. These themes were supplemented by some of the men's identified alternative narratives of 'positive growth' through acceptance from others and themselves following injury. Conclusions: The identified narrative themes provide an understanding of the individual's sense of self and sources of emotional distress, including stigma for men following TBI.
\end{abstract}

Correspondence: Anita Freeman; anita_freeman@hotmail.co.uk 


\title{
Climb Every Mountain - a Road to Recovery
}

\author{
Easton, Ava ${ }^{1}$ \\ ${ }^{1}$ The Encephalitis Society, Malton, UK
}

\begin{abstract}
$B$ ackground and aims: Along with more medical models of rehabilitation there is an opportunity for therapeutic outcomes involving one's peers and acquired brain injury support organisations. This presentation aims to describe examples of successful peer support provided through the voluntary sector. Method: This presentation is based on recorded and observational data (video and written) from a week long trek of Transylvania (during 2011) with 18 people, most of whom were individuals with acquired brain injury or their family members. The participants were users of two national UK voluntary sector providers specialising in acquired brain injury. Results: The paper will suggest that recovery following brain injury can be achieved in settings other than, and in addition to, those offered in clinical and therapeutic settings. Specifically, the paper describes the positive outcomes of peer and voluntary sector support as well as the issues influencing the benefits of this support. Conclusions: Innovative solutions are needed for long-standing and complex problems, such as ongoing support for brain injured populations when they return to their communities. A greater partnership between statutory and peer support and voluntary sectors may be one way to address this.
\end{abstract}

Correspondence: Ava Easton; ava@encephalitis.info

\section{SESSION 11: COGNITIVE RESERVE AND BRAIN STIMULATION}

\section{Does Cognitive Reserve Moderate the Role of Aging in Cognitive Aging?}

Constantinidou, Fofi' ${ }^{1}$; Giogkaraki, Erasmia ${ }^{1}$ and Michaelides, Michael ${ }^{2}$

${ }^{1}$ Center for Applied Neuroscience \& Department of Psychology, University of Cyprus, Nicosia, Cyprus

${ }^{2}$ Department of Education Sciences, European University of Cyprus

$B$ ackground and Aims: Cognitive Reserve (CR) is considered the mind's resilience to neuropathology and has gained attention in recent years in respect to possible contribution in delaying the symptoms of pathological aging. The purpose of the study was to examine the cognitive reserve (CR) hypothesis in association with age, verbal episodic memory (VEP), and executive functioning $(\mathrm{EF})$ in neurologically healthy older adults. A latent variable model for CR was implemented to test the hypotheses stating that CR plays a moderating role on the relationship between age and cognitive functions in healthy aging. Method: A neuropsychological battery was administered to a community-based sample of 253 healthy Greek-Cypriot older adults (Mean age $=75.12, S D=6.19 ;$ Mean years of education $=6.96, S D=4.11)$. Through confirmatory factor analyses we validated latent constructs representing two neuropsychological domains (VEP and EF) and a measure of CR. The CR latent construct had three reflective indicators: education, passive vocabulary, and reading performance. Two structural equation models (SEM) were evaluated. Results: The first SEM tested the direct relationships between age and two latent variables reflecting VEP and EF. Results showed adequate model fit and yielded negative associations among age and both cognitive domains. The second SEM which included CR as a moderator also had an acceptable fit. Results demonstrated that CR strongly moderated the relationship between age and cognitive functions as following: (i) the strength of the (negative) relationship between age and episodic memory was reduced; (ii) the direct 
path from age to executive functions was no longer statistically significant and (iii) all indirect paths were statistically significant. Conclusions: Our findings support the cognitive reserve hypothesis and its moderator effect on the relationships of age on VEP and EF in normal aging. Implications for the theoretical understanding of cognitive reserve on healthy and pathological aging are discussed. It is possible that high levels of CR can help delay the onset of observed clinical symptomatology in adults with mild cognitive impairment.

Correspondence: Fofi Constantinidou; fofic@ucy.ac.cy

\title{
Lateralized prefrontal current-stimulation effects during verbal working memory high-load maintenance
}

Meiron, Oded ${ }^{1}$ and Lavidor, Michal ${ }^{1,2}$

${ }^{7}$ The Gonda Multidisciplinary Brain Research Center, Bar Ilan University, Ramat Gan, Israel

${ }^{2}$ Department of Psychology, University of Hull, UK

\begin{abstract}
$B$ ackground and aims: Cognition enhancing effects have been sporadically observed in humans receiving transcranial direct current stimulation (tDCS) over the prefrontal cortex (Frengi et al., 2005, Jo et al., 2009). Specifically, recent findings demonstrated that on-line left dorsolateral prefrontal (DLPFC) anodal stimulation may improve working memory (WM) performance compared to sham stimulation of the same area (Andrews et al., 2011). We investigated a specific prefrontal verbal WM mechanism, executive attention, and how its function may be affected by WM load, unilateral DLPFC stimulation, and gender, as previous studies showed gender-dependent brain activation during verbal WM tasks. Method: We measured "online" WM performance (accuracy scores) while participants ( $N=41,22$ females) received active or sham (placebo) transcranial direct current stimulation, and implicit learning performance (cued recall accuracy) in a post-stimulation WM task. Online WM and implicit learning performance was assessed using a verbal $n$ Back task (10 min long). Results: Significant lateralized "online" stimulation effects were found only in the highest WM load condition revealing that males benefit from left DLPFC stimulation, while females benefit from right DLPFC stimulation. High WM load performance in the left DLPFC stimulation was significantly related to post-stimulation recall performance. Conclusions: We believe that our findings support the possibility of using brain stimulation to enhance WM in neurological and psychiatric populations, as well as demonstrating novel gender-specific properties related to executive attention, a critical prefrontal core-mechanism of working memory capacity.
\end{abstract}

Correspondence: Oded Meiron; neuron.o.d.m@gmail.com

\section{SESSION 12: MEMORY AND EXECUTIVE FUNCTIONS}

\section{Striving for an Ecologically Valid Assessment of Prospective Memory}

Baylan, Satu ${ }^{1,2}$; Scott, Fiona ${ }^{1}$; Wood, Andrew ${ }^{1}$; Jansari, Ashok ${ }^{3}$ and Evans, Jon ${ }^{1,2}$

${ }^{1}$ Academic Unit of Mental Health \& Wellbeing, University of Glasgow, Glasgow, UK

${ }^{2}$ Sackler Institute of Psychobiological Research, University of Glasgow, Glasgow, UK

${ }^{3}$ School of Psychology, University of East London, London, UK

$\boldsymbol{R}$ ackground and aims: Deficits in prospective memory (PM) are common after brain injury and often have a detrimental impact on everyday 
functioning. Developing ecologically valid assessments of executive functioning has become an important focus of research, with the Dysexecutive Questionnaire (DEX) being frequently used clinically as a measure of real life executive dysfunction after brain injury. This study investigates the ecological validity of a range of tests, including computerised, virtual-reality and "lifelike" tasks, in assessing real-life difficulties in PM post-brain injury. Method: Thirty eight adults (mean age 47 years, SD 10.4) with acquired brain injury completed the following tests of PM: two simple computerised tests, the "JAAM" (office-based virtual-reality task), the modified Hotel Test (mHT) and the Cambridge Prospective Memory Test (CAMPROMPT). Informant rated DEX questionnaires were also completed. Results: Significant correlations were observed between DEX total scores and the following measures: JAAM PM score $(r h o=-.51)$, number of tasks attempted in the mHT $(r h o=-.45)$, with mHT total PM score showing a more modest correlation (rho=-.34), PM measure from the computerised tasks $(r h o=-.40)$ and CAMPROMPT total score $(r=-.39)$. Conclusions: The majority of PM measures showed a significant association with everyday executive functioning with real-life difficulties being associated with poorer task performance. The complexity and high cognitive demands of the JAAM task may best replicate those encountered in real-life problem-solving settings. Nevertheless, the short duration and seemingly "real-life" quality of the multi-tasking environments of the mHT and CAMPROMPT make them clinically appealing.

Correspondence: Satu Baylan; s.baylan@clinmed.gla.ac.uk

\title{
Clinical and experimental validation of a new computerised multi-element test
}

\author{
Cullen, Breda $^{1,2}$; Brennan, David ${ }^{3}$; Manly, Tom ${ }^{4}$ and Evans, Jonathan ${ }^{1}$ \\ ${ }^{1}$ Mental Health and Wellbeing, Institute of Health and Wellbeing, University of Glasgow, UK \\ ${ }^{2}$ Physically Disabled Rehabilitation Unit, Southern General Hospital, Glasgow, UK \\ ${ }^{3}$ Department of Clinical Physics, Southern General Hospital, Glasgow, UK \\ ${ }^{4}$ MRC Cognition and Brain Sciences Unit, Cambridge, UK
}

$\boldsymbol{R}$ ackground and aims: Goal neglect is a common and debilitating conse3 quence of brain injury. Rehabilitative interventions (e.g. goal management training, periodic alerting) have been shown to reduce goal neglect, but it is not yet known how underlying neural activation might change to reflect this. Existing goal neglect tests are unsuitable for use in neuroimaging studies. We developed a computerised multi-element test (CMET), which requires selfmonitoring in order to switch between four simple games within a limited time. We aimed to establish the validity of the CMET, by measuring correlations with existing clinical tests, and by investigating task-related neural activation in a functional MRI paradigm. Method: (1) Eighteen adults with acquired brain injury $(\mathrm{n}=14$ male; M[SD] age $=34.1[10.5])$ were assessed using the CMET, other tests of goal neglect (Hotel Test; Modified Six Elements sub-test from the Behavioural Assessment of the Dysexecutive Syndrome [BADS]) and tests of reasoning. (2) Twelve healthy adults $(\mathrm{n}=8$ male; $M[\mathrm{SD}]$ age $=34.6[7.6])$ underwent fMRI, during which the CMET was administered under two conditions: self-generated switching and experimenter-prompted switching. Results: Among the clinical sample, CMET performance was positively correlated with both the Hotel Test $(r=0.675, \mathrm{p}=0.003)$ and the BADS Six Elements ( $r=0.568, \mathrm{p}=0.014)$, but not with other demographic or clinical measures. In the healthy sample, fMRI demonstrated significant activation in rostro-lateral prefrontal cortex in the self-generated condition compared with the prompted 
condition (peak 40, 44, 4; $\left.Z_{\mathrm{E}}=4.25, \mathrm{p}_{(\mathrm{FWE} \text { corr })}=0.026\right)$. Conclusions: The CMET is sensitive to goal neglect, and elicits neural activation in prefrontal regions which are known to underpin goal-directed behaviour.

Correspondence: Breda Cullen; breda.cullen@glasgow.ac.uk

\title{
Google Calendar: A memory aid to manage prospective memory deficits following acquired brain injury
}

\author{
Haslam, Catherine ${ }^{1}$ and McDonald, Amanda ${ }^{1}$ \\ ${ }^{1}$ Psychology, College of Life and Environmental Sciences, University of Exeter, Exeter EX4 4QG, UK
}

\begin{abstract}
$B$ ackground and aims: Prospective memory impairment is common following acquired brain injury (ABI) and intervention has proved challenging. The current treatment of choice involves using external memory aids as a method of compensation, with those incorporating active reminders proving most successful. In this paper we report findings of an investigation into the effectiveness of a novel external memory aid, Google Calendar. This aid incorporates active reminders and overcomes some of the limitations associated with existing aids. Method: Twelve participants took part in the study, which incorporated a randomised control crossover within-subjects design. This consisted of a 5-week baseline phase, followed by two 5-week intervention phases, where either Google Calendar or a standard diary were used. Participants identified activities to target during the study, and a family member monitored their success. Results: Participants remembered more intentions when using aids and, of these, Google Calendar was found to be more effective than the diary in enhancing prospective memory performance. Specifically, there was a $15 \%$ increase in the likelihood of participants completing their prospective intentions when using memory aids, and a $40 \%$ rate increase in the likelihood of participants completing their prospective intentions when using Google Calendar than when using a standard diary. Discussion and conclusions: Whilst further research is required to substantiate these initial findings, the present findings highlight the potential of Google Calendar in managing prospective memory deficits following ABI.
\end{abstract}

Correspondence: Catherine Haslam; c.haslam@exeter.ac.uk

\section{The effectiveness of brief goal management training (GMT) and SMS text alerts on psychosocial functioning following brain injury: the Assisted Intention Monitoring (AIM) Trial}

Gracey, Fergus ${ }^{1}$; Wilson, Barbara A. ${ }^{1,2}$; Manly, Tom²; Bateman, Andrew ${ }^{1}$; Fish, Jessica ${ }^{2}$; Malley, Donna ${ }^{1}$; Greenfield, Eve ${ }^{2}$ and Evans, Jonathan J. ${ }^{3}$

${ }^{1}$ Oliver Zangwill Centre for Neuropsychological Rehabilitation, Cambridgeshire, UK

${ }^{2}$ MRC Cognition and Brain Sciences Unit, Cambridge, UK

${ }^{3}$ Institute of Health \& Wellbeing, University of Glasgow, Glasgow, UK

${ }^{4}$ National Institute for Health Research (NIHR) Collaborations for Leadership in Applied Health Research and Care (CLAHRC) for Cambridgeshire and Peterborough, Cambridgeshire and Peterborough NHS Foundation Trust, Cambridge, UK

$\boldsymbol{R}$ ackground and aims: Acquired Brain injury ( $\mathrm{ABI}$ ) affects the ability to 3 make plans and remember to do things. Research suggests Goal Management Training (GMT) and content-free cueing might improve these abilities after ABI. The present study tested whether combining these approaches 
(Assisted Intention Monitoring, AIM) would help people with brain injury achieve more of their intentions in day-to-day life. Method: People with ABI and executive deficits (44\% traumatic, $36 \%$ stroke; mean 7 years post-injury) were randomly allocated to receive AIM or a placebo intervention first in a cross-over trial. The AIM first group received 2-3 hours of GMT and were sent 'STOP!' text messages to their phones. The placebo first group received 2-3 hours of information and neutral text messages. Data were collected over $3 \times 3$ week phases (baseline, intervention 1 and intervention 2). The dependent variable was mean proportion of all daily intentions achieved for each intervention phase. Profile of Mood States (POMS) 'total mental distress' was also measured. Results: Repeated measures analysis of variance showed a significant interaction between group (AIM first; placebo first) and time (post intervention 1; post intervention 2) in favour of the effectiveness of AIM (all intentions: $\mathrm{p}<0.05$; researcher-set tasks $(\mathrm{p}<0.01)$. There was no impact on mood as measured by the POMS. Conclusions: AIM intervention appears to help people with ABI achieve daily intentions. However, the effectiveness of AIM varied across types of intention, with the greatest differences arising for researcher-set tasks. Hypotheses regarding this finding will be considered and implications for rehabilitation provision discussed.

Correspondence: Fergus Gracey; fg290@medschl.cam.ac.uk

POSTERS - TUESDAY - Rehabilitation, treatment and management approaches

\section{Early and intensive Occupational Therapy (EIOT) for Delirium Prevention in Older Patients (OP) Admitted to Critical Care Unit (CCU)}

Garrido, Maricel'; Tobar, Eduardo; González Francisco, Briceño Constanza1; Gallegos,

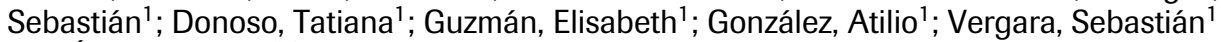
and Álvarez, Evelyn ${ }^{1,2}$

${ }^{1}$ Hospital Clınico Universidad de Chile, Santiago. Chile

${ }^{2}$ Escuela de Terapia Ocupacional Universidad de Chile, Santiago. Chile FONIS SA10I20015

$\boldsymbol{R}$ ackground and aims: Delirium is a complication in older patients (OP); $\mathcal{B}$ it has an incidence rate of $70-87 \%$ in critical care unit (CCU). Therefore, it increases mortality, hospital stay, hospital cost, and cognitive impairment. Occupational Therapy (OT) improves independence at discharge and reduces delirium in patients undergoing mechanical ventilation. The present study compares the efficacy of non-pharmacological standard prevention (control group) versus non-pharmacological standard prevention plus Early and Intensive Occupational Therapy (EIOT; Intervention Group) in the delirium duration in OP admitted to CCU. Method: A randomized clinical trial involving 70 OP admitted to CCU for monitoring of acute or chronic illness, without signs of cognitive impairment and delirium at admission. The Control Group received reorienting, early mobilization, correction of sensory deficits, environmental management, protocol for sleep and reduction of drugs. The Intervention Group additionally received multisensory stimulation, positioning, cognitive stimulation, training in activities of daily living, motor stimulation of the upper extremities and family participation. Each patient in the EIOT undertook two daily sessions of 40 minutes with an OT for 5 days. Patients were assessed for delirium with the Confusion Assessment Method (CAM) and Delirium Rating Scale (DRS) in each session. Level of functional independence (FIM), strength of grip (Jamar Dynamometer) and cognitive status (Mini Mental Status Exam) 
were assessed at discharge. Results: Participants in the EIOT intervention showed lower incidence of delirium compared to the control group (16.1\% versus $3.1 \%)$, but the delirium duration (days) was similar $(2.3+1.3$ versus 3.0). The EIOT intervention also had higher FIM-motor scores (46.5 versus $58.3, p=0.03$ ) and a shorter hospital stay (20.6 days versus 10.4, $p=.009$ ). Conclusion: The findings support that EIOT is effective in reducing delirium in hospitalized OP, and may reduce their stay and maintain levels of functional independence at discharge.

Correspondence: Evelyn Álvarez; evalvarez@med.uchile.cl

\title{
Effects of working memory training on functioning in daily life
}

\author{
Björkdahl Ann 1,2,3; Esbjörnsson Eva1; Svensson Siv ${ }^{4}$ and Åkerlund Elisabeth ${ }^{4}$ \\ ${ }^{1}$ Gothenburg University, Sahlgrenska Academy, Institute for Neuroscience and Physiology, Rehabilitation \\ medicine, Sweden \\ ${ }^{2}$ Ersta Sköndal University College, Campus Bräcke, Sweden \\ ${ }^{3}$ Sahlgrenska University Hospital, Physiotherapy and occupational therapy department, Sweden \\ ${ }^{4}$ Sahlgrenska University Hospital, Rehabilitation medicine, Sweden
}

\begin{abstract}
$B$ ackground and aims: Working memory (WM) problems are common after brain injury and have great implications for functioning in daily life. The aim of the study was to determine whether computerized working memory training improves WMand has an impact on activity and participation. Method: The study was randomized with an intervention group ( $\mathrm{IG}, \mathrm{N}=20$ ) and waiting list control group $(\mathrm{CG}, \mathrm{N}=18)$, and examined the effects of a WM training program for patients with brain injury who were aged 18-65 years andadmitted for outpatient rehabilitation. Assessments were made at baseline (A1), 6 weeks (A2) and 18 weeks (A3). Between weeks 18 and 24 (A4) the CG was offered the WM training and were then assessed at 24 weeks. Assessments included tests of cognitive functioning (Barrow Neurological Institute Screening for higher cortical functions [BNIS], WAIS-III-NI subscale Working Memory [WM], Rivermead Behavioral Memory Test [RBMT]) and occupational performance (Assessment of Motor and Process skill [AMPS]) and questionnaires on fatigue (Fatigue Impact Scale [FIS]), depression and anxiety (Hospital Anxiety and Depression Scale [HADS]). Result: Both groups improved on WM between A1 and A2 (IG $p=0.000$, CG $p=0.003$ ) and the IG also improved on the BNIS $(p=0.005)$ and FIS $(p=0.038)$. After training (A2-A3) the only improvements were seen in AMPS motor skill for the IG $(p=0.038)$. The CG that trained between $\mathrm{A} 3$ and $\mathrm{A} 4(n=8)$ improved on WM $(p=0.012)$ and everyday memory problems $(\mathrm{RBMT}, \mathrm{p}=0.042)$. Between A1 and A3 both groups improved on AMPS motor skills (IG $p=0.016$, CG $p=0.013$ ). The training also had a significant impact on depression for both groups (IG $p=0.003$, CG $p=0.024)$ and on anxiety in the CG $(p=0.017)$. Conclusion: Although changes in WM were evident in both groups, the training seems to have had a generalized effect on occupational performance and also improved mood. The IG also experienced less fatigue after the training.
\end{abstract}

Correspondence: Ann Björkdahl; ann.bjorkdahl@esh.se 


\title{
The use of movement sensors and automated voice prompts to address orientation and nocturnal wandering in an individual with acquired brain injury
}

\author{
Brown, Pamela'; Findlay, Gemma ${ }^{1}$; Goodfellow Rebecca ${ }^{1}$ and O'Neill, Brian ${ }^{1}$ \\ ${ }^{1}$ Brain Injury Rehabilitation Trust, Graham Anderson House, 1161 Springburn Road, Glasgow, G21 1 UU, \\ Scotland, UK
}

\begin{abstract}
$B$ ackground and aims: Impaired orientation and awareness are common consequences of severe acquired brain injury which can lead to nocturnal wandering and confer risk and distress. This single case study used movement sensors and movement-activated voice prompts to reduce the number of times an individual with disorientation got up during the night. Method: $\mathrm{M}$ is a 62 year-old single man with a 6 month history of intracerebral haemorrhagic stroke. He had persisting severe cognitive difficulties in memory, executive functioning and visual perception. In an inpatient rehabilitation setting he had regular night-time wandering with confusion, topographic disorientation and apparent distress and irritability when approached. In this ABA design we used movement sensors (Just-Checking) to monitor activity between 0:00 and 7:00. A movement-activated voice prompt (Wander-Alarm) was introduced to reassure and direct him to the bathroom or encourage return to bed. Randomisation statistics will be reported. Results: During the baseline period M moved around (or left) his bedroom at night 166 times in 24 days (average $=7$ times per night; ranging from 1-16 times). A movement-activated voice prompt was then introduced. During the intervention period there was a total of 30 movement recordings (average $=3.8$ times per night; range $2-6$ ). Conclusions: Preliminary data demonstrates a reduction in recorded nocturnal wandering episodes due to the intervention. This will form part of a larger case-series.
\end{abstract}

Correspondence: Pamela Brown; pamela.brown@thedtgroup.org

\section{Is errorless learning effective for retrieval deficits? A single-subject experimental design}

$\overline{\text { Covre, Priscila }^{1,2} \text {; Landucci, Daniella }}{ }^{2}$; Bolognani, Silvia Adriana Prado Landucci, Daniella ${ }^{2}$ and Bueno, Orlando Francisco Amodeo Bolognani ${ }^{1,2}$

${ }^{1}$ Departamento de Psicobiologia; Universidade Federal de Sao Paulo; Sao Paulo; Brazil;

${ }^{2}$ Centro Paulista de Neuropsicologia; Associaçao Fundo de Incentivo a Pesquisa; Sao Paulo; Sao Paulo; Brazil

\begin{abstract}
$B$ ackground and aims: Errorless learning techniques (ELT) have been shown to be effective in teaching new information to individuals with severe memory problems, but are they effective in transforming inconsistent retrieval of already learned facts into more consistent recollections? In this single case study, we explored the effectiveness of ELT for both conditions. Methods: A 56 year-old man with profound memory and executive functions impairments was referred to our clinics four years after suffering a post-anoxic encephalopathy due to an episode of cardiac arrest. The patient was anosognosic and very inconsistent in recognizing and remembering family members (sons, daughter, wife and grandson). The family's rehabilitation goals were for him to learn what happened to him, about his memory problems and to remember his family members more consistently. Results: Baseline data were collected once a week for 5 weeks before treatment; mean correct answer + SD for self and family questions were $0.15+0.11$ and $0.68+0.11$, respectively. The patient underwent 13 weekly sessions of rehabilitation where ELT were used to teach him about self and family facts. The same questions used at baseline
\end{abstract}


were asked at every session. Results for the last 5 sessions ( self $=0.71+0.32$; family $=0.69+0.06$ ) revealed that the patient was able to partially learn facts about himself, but recollection of family members had not improved. Further, the patient did not consistently remember the new facts. Conclusion: Overall, ELT were not effective for executive retrieval deficits.

Correspondence: Priscila Covre; priscilacovre@gmail.com

\title{
Do Technological Memory Aids Support Abilities or Activities? Conceptual Aspects to be Considered Regarding the Effectiveness of Technological Aids to Support Prospective Memory Failures
}

\author{
Cruz, Gabriela ${ }^{1,2}$; Goudie, Nicola ${ }^{2}$ and Petrie, Susan ${ }^{2}$ \\ ${ }^{1}$ School of Psychology,University of Glasgow, Glasgow, Scotland, UK \\ ${ }^{2}$ Community Treatment Centre for Brain Injury, Glasgow, Scotland, UK
}

\begin{abstract}
$B$ ackground and Aims: Occupational performance (OP) has many levels of organization, including the top level (re-engagement in meaningful activities) and lower levels corresponding to activities and tasks, which require cognitive abilities such as prospective memory (PM). PM is the ability to execute an intended action after a delay. Technology is increasingly used to support PM; however, it is not always effective. This paper aims to analyse effectiveness of a technological memory aid in relation to levels of organization of the OP. Method: Three participants with brain injury were trained in the use of 'Google Calendar' including how to set up SMS notifications to prompt activities. Target activities were identified on the Canadian Occupational Performance Measure (Law, 2005) through each participant's perception of the importance and difficulty in engaging in meaningful activities. Results: Two of the three participants perceived improvements in their abilities and showed an objective increase in independent performance. A critical factor identified appeared to be motivation for achieving the intended action. Conclusion: The memory aid presented operates by supporting specific cognitive abilities. The activity provides the context for the development of the ability, and it brings together other aspects that need to be considered, such as the meaningfulness of the activity and motivation for using a technological memory aid.
\end{abstract}

Correspondence: Gabriela Cruz; g.cruz@psy.gla.ac.uk

\section{Differential effects of visual imagery strategies for prospective memory perfor- mance in a single case study of hydrocephalus}

\author{
Edginton, Trudi $^{1}$ and Raval, Pooja ${ }^{1}$ \\ ${ }^{1}$ Department of Psychology, University of Westminster, London, UK
}

$\boldsymbol{B}$ ackground and aims: Hydrocephalus is associated with a unique pattern $\boldsymbol{B}$ of cognitive strengths and weaknesses that are further exacerbated by impairments in level of subjective awareness (Iddon et al, 1996; 2001; 03; 04; Loveday and Edginton, 2010). Objective prospective memory (PM) deficits can impact greatly on everyday functioning by disrupting the ability to remember and enact delayed intentions. This study aimed to explore the benefits of visual imagery strategies that may augment binding processes that underlie prospective memory functioning and improve insight in a single case study design. Method: We report the case of KW, a 39 year old female with hydrocephalus who reports subjective cognitive difficulties. Using a single case design with 
matched healthy controls (HCs $n=5$ ) we compared visual imagery and PM performance using the VVIQ (Marks, 1973), the Clock task (Grossi et al, 89) and the Letter Forms Test (Weber \& Castleman, 1970). Subjective prospective memory difficulties were assessed with the PRMQ (Smith et al, 2000) and time-based and event-based prospective memory performance was assessed, with and without directed visual imagery, using the CAMPROMPT (Wilson et al., 2005). Results: Results revealed significant differences in PM performance with impaired event-based PM in KW $(t(4)=-3.693, p<0.01)$ in contrast to comparable self-report on the PRMQ with HCs $(t(4)=0.755, p=0.246)$. There was a differential effect of directed imagery with improved performance for $\mathrm{KW}$ for event-based PM, restoring performance to a comparable level to the HCs. Conclusions: Targeted visual imagery strategies can improve PM performance in an individual with hydrocephalus in line with healthy controls. The benefits of visual imagery strategies appear to be isolated within eventbased PM supporting the importance of binding processes in event-based PM (Gonneaud et al, 2011). Successful implementation of cognitive strategies can also contribute to increased awareness of executive function impairments.

Correspondence: Trudi Edginton; t.edginton@westminster.ac.uk

\title{
Integrated Interdisciplinary Team (IDT) approach between anthroposophic and conventional medicine: an effective rehab treatment programme in the recovery of a vegetative state patient following tuberculosis meningitis
}

\author{
Enteria, Reymund ${ }^{1}$ and Florschutz, Gerhard ${ }^{1}$ \\ ${ }^{1}$ Occupational Therapy Department, The Raphael Medical Centre, Tonbridge, UK
}

$B$ ackground and aim: The case of I.M a 28 year old woman admitted for treatment following tuberculosis meningitis (TBM) is presented. In a vegetative state (VS) on admission, I.M went on to make a good recovery after receiving anthroposophic and conventional intervention from an interdisciplinary team. She is now walking and functioning independently. This paper aims to report the recovery process. Method: The anthroposophic and conventional treatment administered is described and data are presented from the various assessments and treatments administered from admission to the present. Photographs and videotapes provide visual evidence of I.M's recovery. Outcome measures include the JFK coma recovery scale and the FIM/FAM. Results: After receiving more than 2 years of integrated rehabilitation, I.M. is now ready for discharge to home. The FIM/FAM scores reflect the change from VS to near independence. She remains with memory difficulties and left sided weakness. The recovery process of an individual from VS to independence (with some support) followed specific stages and occurred after various treatment approaches and flexible therapy programmes. Different members of the interdisciplinary team were involved in collaboration with the patient and/or family members to meet the needs of the patient in achieving her goals. Conclusion: When recovery from VS occurs, the process and length of recovery varies and may take a long time. Treatment efforts and hope need to be maintained for patients in VS following TBM, given that recovery is considered feasible. An integrated approach combining anthroposophic and conventional medicine was found, in this case, to be effective.

Correspondence: Reymund Enteria; ot@ raphaelmedicalcentre.co.uk 


\title{
EMDR-treatment of trigeminal neuralgia pain in a stroke patient: $A$ case study
}

Groet, Erny ${ }^{1}$

${ }^{1}$ Rehabilitation Centre Heliomare, Wijk aan Zee, The Netherlands

\begin{abstract}
$B$ ackground and aims: Trigeminal neuralgia is a neuropathic disorder, characterized by episodes of intense pain in the face. The pain may not always respond to medication. This case study aimed to describe the treatment of trigeminal neuralgia for a 39 year old man who experienced a stroke that resulted in cognitive impairments, an emotional disorder, fatigue and extreme facial pain. In addition to receiving cognitive rehabilitation, treatment focused on pain reduction. Method: In the first year he received carbamazepine, without any effect. He then received 7 weekly sessions of Eye Movement Desensitization and Reprocessing (EMDR) treatment that focused on his facial pain. The patient kept a record of his pain level twice a day for the following periods: 3 weeks prior to EMDR-treatment, during the whole treatment period and 6 months post treatment. In accordance with the EMDR-protocol, the pain rating was requested by the therapist right before, during and after each treatment session. Results: The EMDR-treatment was associated with a reduction in self-reported pain level from an average of 6 (on a 10 point scale) at pre-treatment to an average of 0.5 at post treatment. Conclusion: Medical treatment for trigeminal neuralgia is not always sufficient to reduce the pain. This case study shows that EMDR might be helpful for reducing pain caused by trigeminal neuralgia.
\end{abstract}

Correspondence: Erny Groet; e.groet@heliomare.nl

\section{Come dine with me: shopping and cooking versus formal therapy for severe dys- phasia following traumatic brain injury}

Hinchcliffe, Angela ${ }^{1}$

${ }^{7}$ Raphael Medical Centre, Hildenborough, UK

$\boldsymbol{R}$ ackground and aims: The nature of communication disorders following $\mathcal{B}$ traumatic brain injury is rarely straightforward. Individuals often present with a combination of disorders and complicating factors which need to be recognised to enable the process of recovery. This paper aims to highlight the benefits of working in a functional and imaginative way by describing the progress of an individual (UV) who presented with severe dysphasia and other complicating factors following a severe brain injury. Method: A descriptive case study is presented on UV, who has made considerable progress since the time of her injury. She has a severe receptive and expressive dysphasia together with cognitive and emotional difficulties including extreme anxiety. Her progress and the multi-disciplinary collaboration involved in her rehabilitation will be discussed and compared with formal speech and language therapy test results. Results: UV was assessed during the course of treatment by formal tests such as the Frenchay Aphasia Screening Test and the picture naming test of the Psycholinguistic Assessment of Language Processing in Adult Acquired Aphasia. Results from these tests of language do not show the level of progress which is apparent in her functional activities. Discussion: The reasons for the discrepancy between test results and functional progress will be discussed in relation to UV's individual needs and characteristics. Conclusion: Progress can be realised in very different ways and the importance of adopting 
a functionally relevant approach to rehabilitation is especially important for individuals with multiple complex needs.

Correspondence: Angela Hinchliffe; angi.hinchcliffe@ tiscali.co.uk

\title{
A multi-centre controlled pilot study to evaluate a telemedicine system for the assisted living of people with dementia and their carers
}

\author{
Jahanshahi, Marjan ${ }^{1}$; Torkamani, Mariam ${ }^{1}$ and McDonald, Louise ${ }^{1}$ \\ ${ }^{1}$ Cognitive Motor Neuroscience Group, UCL Institute of Neurology \& the National Hospital for Neurology \& \\ Neurosurgery, London, UK
}

\begin{abstract}
$B$ ackground and aims: Estimates show that there are 35.6 million people with dementia (PwD) worldwide. This prevalence rate is expected to rise with increased life expectancy. This could pose a challenge to health systems, so new ways of managing dementia need to be considered. Around $40 \%$ of PwD reside in care homes, costing over $\$ 315$ billion worldwide, and the $60 \%$ in the community are mainly supported by informal carers. The aim of this project was to evaluate 'telemedicine' for remote monitoring of the cognitive and behavioral state of $\mathrm{PwD}$ and detection of carer burden, a principal determinant of nursing home placement of PwD. Method: 60 PwD (MMSE $\geq 11$ and Barthel Index $\geq 35$ ) living at home with a full-time carer were recruited in three pilot sites in the UK, Spain and Greece, and were randomly allocated to the telemedicine group $(n=30)$ or the control group $(n=30)$. Assessments of the PwDs' cognition, mood and quality of life, and the carers' burden, mood and quality of life were carried out at baseline, 3 and 6 months. Results: The results of the project are being analysed. It is predicted that increased communication and availability of information through telemedicine will enhance detection of change, management of the symptoms of PwD, and provide support for the carers relative to the control group. Discussion and conclusion: If positive, the outcomes can be a step forward towards better management of PwD living at home and avoid/delay institutionalisation, through frequent remote monitoring of their symptoms and supporting their carers.
\end{abstract}

Correspondence: Marjan Jahanshahi; m.jahanshahi@ucl.ac.uk

\section{Effect of attention rehabilitation on cognitive functions in patients with schizophre- nia}

\author{
Kim, Myung-Sun Kim ${ }^{1}$ and Jang, Hui-Jin ${ }^{1}$ \\ ${ }^{1}$ Department of Psychology, Sungshin Women's University, Seoul, Korea
}

$B$ ackground and aims: Attention deficit has been regarded as a core symptom of schizophrenia. Impaired cognitive functions affect patients' social functions more negatively than schizophrenic symptoms, and it has been reported that cognitive rehabilitation improves cognitive function and reduces the symptoms (Bell et al., 2009). The aim of this study was to examine whether attention rehabilitation could improve the cognitive functions in people with chronic schizophrenia Method: Forty patients with schizophrenia were assigned to one of three groups; experimental $(n=13)$, comparative $(n=13)$ and control $(n=14)$ groups. The attention training (Bracy, 1994) and Korean typing program were administered to experimental and comparative groups, respectively. No training was given to the control group. Neuropsychological tests and the Positive and Negative Syndrome Scale (PANSS) were 
administered before and after attention training. Results: The three groups did not differ on performance on neuropsychological tests and PANSS prior to training. The experimental group showed improved performance on the copy $(t=-4.74, p<.01)$ and delayed-recall $(t=-6.90, p<.01)$ of Rey Osterrieth Complex Figure Test and Stroop color-word interference $(t=2.64, p<.05)$. They also showed reduced negative $(t=2.91, p<.05)$ and general pathological symptoms $(t=4.31, p<.01)$ after training; however, the other two groups did not show these changes. Conclusions: These results indicate that attention rehabilitation is effective for improving cognitive functions of people with schizophrenia, and that cognitive rehabilitation is important for the treatment of schizophrenia

Correspondence: Myung-Sun Kim; kimms@sungshin.ac.kr

\title{
Intensive Cognitive Rehabilitation (ICR): preliminary results indicate enhancement of cognitive function and individual goal attainment
}

\author{
Kjeverud, Anita ${ }^{1}$ \\ ${ }^{1}$ Unit of Physical medicine and rehabilitation, Innlandet Hospital Trust, Norway
}

\begin{abstract}
$B$ ackground and aims: Cognitive rehabilitation aims to enhance cognitive function, insight and quality of life in individuals with cognitive dysfunction. The Unit of Physical Medicine and Rehabilitation at Sykehuset Innlandet is currently offering a programme of Intensive Cognitive Rehabilitation (ICR) based on Malia and Brannagan's Brain Tree-model. As part of an ongoing evaluation of the programme, the preliminary results from the rehabilitation unit at Ottestad will be discussed. Method: The programme of ICR runs over 14 weeks, and involves alternating between working as a group in the institution (4 weeks) and working on personally relevant goals and cognitive training at home (10 weeks). The programme consists of education, training of specific cognitive functions, training in the use of compensatory strategies, practice in relevant daily activities, daily physical exercise and goal-setting. Results: Ten participants have completed the programme and six have received neuropsychological testing before and at the end of the 14 week programme. Each had different profiles of cognitive strengths and deficits, and all six participants showed improvement in some domain. All participants showed improvement on tests of memory and all achieved at least one of their personal goals. Further, five participants have returned to work-related activities. Conclusion: The ICR programme is still under development; however, promising results are evident to date in respect to personal goal attainment and improvement in cognitive function as measured by neuropsychological tests. According to feedback from the participants, a key factor related to these outcomes is the alternation between staying in the unit as a group and working towards goalattainment at home.
\end{abstract}

Correspondence: Anita Kjeverud; anita.kjeverud@sykehuset-innlandet.no

\section{Using guided affective imagery in psychotherapy of patients with amnestic syn- drome as a result of traumatic brain injury}

\author{
Daminov, Vadim $^{1}$ and Kulikova, Irina ${ }^{1}$ \\ ${ }^{7}$ Neurorehabilitation Department, Pirogov National Medical Surgery Centre, Moscow, Russia
}

$\boldsymbol{B}$ ackground and aims: Traumatic brain injury (TBI) and amnestic syndrome usually cause psychological trauma, anxiety and depression. The 
respective cognitive deficits lead to procedural difficulties in psychotherapy sessions and narrow the range of psychotherapy methods available. The aim of this study was to investigate the suitability of guided affective imagery (katathym imaginative psychotherapy) in psychotherapy for patients with amnestic syndrome. Methods: 24 patients (17-48 years) with amnestic syndrome of a variable degree after TBI and apparent psychological problems. Patients with sufficiently intact perception and in the late rehabilitation period were involved. Diagnostic methods included the Luria neuropsychological battery. The psychotherapy course included 8-16 sessions conducted 1-2 times a week. The basis of psychotherapy was fantasy generation, which was quite possible for these patients because it does not place demands on memory recall or retrieval of personal biographical experience (including the content of psychotherapy sessions). The patients chose and worked on one of the main motives of imagery reflecting in the best way their inner psychodynamic state. Such work accessed and resolved emotional conflicts that operated at subconscious level. Results: The described methods were shown to be effective, with patients reporting lower levels of depression and anxiety and improved use of coping resources. A key finding was that the effects of guided imagery were evident even for patients with significant memory deficit. Conclusion: Guided affective imagery appears to be a suitable approach to use in psychotherapy for patients with amnestic syndrome.

Correspondence: Irina Kulikova; i_kulikova@inbox.ru

\title{
Can alexithymia be improved? A case study
}

\author{
Prince, Leyla ${ }^{1}$ and Longworth, Catherine ${ }^{2}$ \\ ${ }^{1}$ Speech and language Therapy, The Oliver Zangwill Centre, Ely, UK \\ ${ }^{2}$ Clinical Psychology, The Oliver Zangwill Centre, Ely, UK
}

$\boldsymbol{R}$ ackground and aims: Defined as an inability to identify and describe $\mathcal{B}$ one's emotions verbally, individuals with alexithymia have shown reduced responsiveness to psychotherapy (Wood, 2010). Prevalence in the TBI population is high $(57 \%)$ and often associated with co-morbidity. The Toronto Alexithymia Scale (TAS) is a widely used, reliable and validated measure. This single case study investigated whether (a) alexithymia can improve through intervention, and (b) whether the TAS is sensitive to any change. Methods: A single case experimental design was employed, involving a 32 year old female attending holistic rehabilitation. NB presented eight years post injury (subarachnoid haemorrhage), further complicated by a complex pre-injury history. The TAS was administered before and after intervention. Aims were to increase awareness and expand existing semantic concepts through language for six basic emotions. Intervention was carried out weekly with a speech and language therapist, alongside psychotherapy sessions in which discursive items were monitored. NB conducted daily self-monitoring throughout, along with Actiheart monitoring. Results: TAS scores changed from 72 to 54, suggesting a marked positive change in NB's perception of her awareness of and descriptive ability in relation to emotion. Qualitative analysis of discursive items noted a shift in narrative from a physical focus to an emotional focus. Programme outcomes showed similar improvements in depression and selfesteem and a perceived reduction in symptoms. Conclusion: Preliminary findings suggest that alexithymia can be improved within the context of a holistic 
rehabilitation programme. The TAS was found to be sensitive to change as an outcome measure.

Correspondence: Leyla Prince; Leyla.prince@ozc.nhs.uk

\title{
The role of the speech and language therapist in working with couples in Neurore- habilitation
}

\author{
Prince, Leyla ${ }^{1}$ \\ ${ }^{1}$ Speech and language Therapy, The Oliver Zangwill Centre, Ely, UK
}

\begin{abstract}
$\boldsymbol{R}$ ackground and aims: Family therapy is usually delivered by systemic ther3 apists, counsellors or psychotherapists. Speech and language therapists' (SLT) work with families typically focusses on the communication difficulties of the person with brain injury. This role has expanded at the Oliver Zangwill Centre, where the SLT has become increasingly involved in supporting families with interpersonal difficulties from a communication perspective. This study discusses the findings observed over a series of cases. Method: This is a descriptive study of non-consecutive cases. Three couples, where one partner is brain injured, were seen alongside holistic neurorehabilitation. Sessions aimed at addressing communication difficulties in interpersonal interactions. A process adapted from the Y-shaped model of rehabilitation was applied (Gracey et al, 2009); identifying the discrepancy in interactional styles, developing a shared formulation of difficulties and identifying what can be changed. Cognitive Behavioural Therapy techniques were used to facilitate change. Feedback was retrieved verbally in a reflective group session. Results: Having time to build a shared understanding of difficulties and the discrepancy post-injury was valuable even if this did not lead to change. Focussing on communication allowed for the introduction of strategies, but also acted as a catalyst for revealing more salient issues. Cognitive behavioural therapy techniques were useful, although compliance was an issue. Conclusion: SLT brings a different perspective to understanding interpersonal difficulties in family work. The SLT intervention and psychotherapy can be mutually complimentary and improve efficacy of family work, and disperses responsibility for family work to more members of a treating team.
\end{abstract}

Correspondence: Leyla Prince; Leyla.prince@ozc.nhs.uk

\section{Can an out-patient rehabilitation program help patients with acquired brain injury to return to work?}

\author{
Ryman, Mikael ${ }^{1}$; Sareneva, Eija ${ }^{1}$ and Bohlander, Frans ${ }^{1}$ \\ ${ }^{1}$ Department for physical medicine and rehabilitation, Sykehuset Innlandet, Ottestad, Norway
}

ackground and aims: Since 2008, more than 200 patients with acquired
brain injury have taken part in an interdisciplinary rehabilitation program
with focus on returning to work. The major impairments for these patients
were evident in the cognitive domain. Each patient had a case manager who
offered assistance and was in liaison with the work place. Medical, motor and
neuropsychological assessments were a part of the interdisciplinary rehabili-
tation program as well as group programs for patients. Method: 185 patients
were examined who participated in the program. Evaluation schedules from 4
different out-patient groups with a total of 35 patients were analyzed. Results:
More than 50\% had returned to work after attending the program, compared 
to $16 \%$ at the start, and there was a significant reduction in received benefits. Those who had started the program after a shorter sick-leave had better return to work outcomes. Patients reported a better understanding of the consequences of brain injury and that they were more realistic in relation to work and in other areas. Meeting others in the same situation was viewed as an important part of the group. Conclusions: Participation in an early out-patient rehabilitation intervention for patients with acquired brain injury appears to contribute to positive return to work outcomes. The experience of meeting others with an acquired brain injury was a particularly valued part of their rehabilitation.

Correspondence: Mikael Ryman; Mikael.Ryman@sykehuset-innlandet.no

\title{
Art as therapeutic recreation following acquired brain injury (ABI) to enhance emotional regulation
}

\author{
Sainsbury, Sally ${ }^{1}$ and Lee, Kirsty ${ }^{1}$ \\ ${ }^{7}$ Allied Health, Brightwater Oats Street Rehabilitation Facility, Perth, Australia
}

\begin{abstract}
$B$ ackground and aims: Emotional dysregulation and psychological disturbance are common consequences of acquired brain injury (ABI). An art therapy group was conducted which aimed to provide participants with a structured therapeutic experience, and encourage emotional expression and foster independence. Positive intended emotional effects included improved selfesteem and motivation and a reduction in anxiety, depression and destructive behaviours. Furthermore, the art therapy group was designed to provide opportunities for social inclusion and self-reflection and to offer artists a context to redefine their post-trauma artistic style. This paper aims to describe preliminary outcomes of the therapy group. Method: 6 participants with ABI in a slow-stream residential rehabilitation setting took part in 6 weeks of group art therapy. The sessions were held once per week for 90 minutes. One participant had been an accomplished visual artist prior to the ABI. Three had experienced clinical depression post ABI, including suicidal ideation. Two other individuals had emotional lability, with one having aggressive outbursts. Results: Qualitative data in the form of participant surveys, anecdotal evidence and quality-of-life measures were collected prior to, during, and after the program. These measures showed an overall positive trend towards increased emotional regulation and improved sense of well-being. Conclusion: While participation in group art therapy appeared to enhance emotional regulation after therapy, the long-term maintenance and generalisation beyond the therapeutic context needs to be examined.
\end{abstract}

Correspondence: Sally Sainsbury; sally.sainsbury@brightwatergroup.com

\section{A case description from Intensive Cognitive Rehabilitation (ICR)}

\section{Skøien, Reidun ${ }^{1}$ \\ ${ }^{1}$ Unit of Physical medicine and rehabilitation, Ambulant Rehabilitation Team, Innlandet Hospital Trust, Norway}

$\boldsymbol{R}$ ackground and aims: The Intensive Cognitive Rehabilitation (ICR) was designed to provide a better follow-up of patients with brain injury who experience cognitive difficulties. This case study aimed to describe one patient's experience of the ICR and her functional outcomes. Method: Anne is a special educator who had a subarachnoid haemorrhage in August 2009. She experienced memory problems and became stressed and tired more easily. In 
January 2010 she was admitted to the Rehabilitation Unit for cognitive assessment. Her aim was to return to work at a 50\% capacity. In February 2010 an Ambulant Rehabilitation Team followed her up. The Team assisted Anne in meetings with The Social Security Unit and within her workplace. In April 2011 she was offered the ICR. Anne had three one-week stays inside the Institution, in April, May and June. In the periods in between she was followed up by the Ambulant Rehabilitation Team at home and at her workplace. Her aim was to continue to work and to learn strategies to improve her memory, sense of time, ability to manage multiple tasks and to be aware of her work capacity. Results: At the start of ICR Anne was capable of concentrating 30 - 45 minutes and needed to rest often. After several weeks she was able to tolerate stress better. At the end of ICR she had improved her ability to process information and had a higher tolerance to noise, but she still required a more supported environment when working longer sequences. She came to realise that working at $25 \%$ of her original position was realistic and felt comfortable with that. Conclusion: At the end of 2011 Anne was satisfied with her work situation, and perceived that her memory had improved with strategy use, although she still experiences difficulty with judging time. A graded pension is currently being organised for her.

Correspondence: Reidun Skøien; Reidun.Skoien@sykehuset-innlandet.no

\section{What are the benefits of Intensive Cognitive Rehabilitation (ICR)}

Skøien, Reidun ${ }^{1}$

${ }^{1}$ Unit of Physical medicine and rehabilitation, Ambulant Rehabilitation Team, Innlandet Hospital Trust, Norway

$B$ ackground: The Rehabilitation Units in the counties of Hedmark and Oppland in Norway started in 2010 as part of a collaboration within ICR for better follow-up of patients with cognitive difficulties (at Ottestad and Gjøvik). The ICR program is partly based on The Brain Tree Training Concept. Professionals associated with ICR are neuropsychologists, neurologists, nurses, physiotherapists, occupational therapists, speech therapists and social workers. A core group has overall responsibility for the ICR program. Aims: The aim is to train patients to improve cognitive function in daily life, at home and at work. Method: ICR runs over 3-4 months with four one-week intensive trainings inside the institution with weeks at home and at work in between. Key components are training, establishing strategies and routines, and getting support in a process over time. An Ambulant Rehabilitation Team cooperates with the Rehabilitation Units at recruitment and at follow-up of patients. Individual aims and a schedule of activities is set up by professionals together with the patient at the start and is evaluated after completing the program. Results: At the end of 2011, a total of 19 patients had completed ICR in both counties. ICR has been found to present the patients with challenges which increased their acknowledgement and insight into their difficulties. The programme also fosters a feeling of "not being alone with my difficulties" and provides someone to talk to that is not a professional. Observations conducted between the two units have resulted in better coordination and practical cooperation, with the exchange of experience and expertise being very useful. Conclusions: Some positive effects of ICR are evident; however, more long-term research and experience in conducting ICR is needed to evaluate the benefits of the programme.

Correspondence: Reidum Skøien; Reidun.Skoien@sykehuset-innlandet.no 


\title{
A multidisciplinary treatment by patients with mild traumatic brain injury
}

Vikane, Eirik ${ }^{1}$ and Skouen, Jan Sture ${ }^{1,2}$

${ }^{1}$ Department of Physical Medicine and Rehabilitation, Haukeland University Hospital, Bergen, Norway

${ }^{2}$ Physiotherapy Research Group, Department of Public Health and Primary Health Care, University of Bergen, Norway

\begin{abstract}
$B$ ackground and aims: Randomized controlled studies have shown conflicting results from early rehabilitation of mild traumatic brain injury (MTBI) patients. Further studies should focus on patients with verified cognitive and psychological problems at the first 1-3 months post injury. The aim of this study was to determine the need for a neuropsychologist in a multidisciplinary team. Method: Patients 16-55 years admitted consecutively to the Neurosurgery Department in Bergen from 2009 to 2011 with MTBI defined according to the Glasgow Coma scale and unconsciousness less than 30 minutes, were offered first a screening examination by a rehabilitation specialist at two months follow-up. Patients on sick-leave and with related symptoms were offered a clinical examination by a multidisciplinary team. Standardized neuropsychological tests detecting brain dysfunctions were performed. Patients were then randomly allocated to a structured multidisciplinary follow-up program or referred back to their general practitioners. Results: Of 411 patients admitted consecutively to the Neurosurgery Department with MTBI, 117 were excluded from the study. 192 attended the follow-up session, and 50 were offered a multidisciplinary examination according to the inclusion criteria. A total of 45 patients $(90 \%)$ had neuropsychological test results indicating brain dysfunction due to the trauma or pre-morbid functioning. Conclusions: Preliminary results from this ongoing study confirm the need for a neuropsychologist in a multidisciplinary team, since the majority of patients had neuropsychological deficits two months post-injury. It remains to be determined whether an outpatient multidisciplinary follow-up model will have a better outcome than ordinary primary health care.
\end{abstract}

Correspondence: Erik Vikane; eirik.vikane@ helse-bergen.no

\section{Benefits of a Therapy Dog in a Holistic Rehabilitation Programme}

Winegardner, Jill ${ }^{1}$; Ashworth, Fiona ${ }^{1}$; Jennings, Caroline ${ }^{1}$ and Wilson, Barbara A. ${ }^{1}$

${ }^{1}$ Oliver Zangwill Centre, Ely, UK

$\boldsymbol{R}$ ackground and aims: The value of therapy animals in populations includ3 ing elderly people, people in prison, and people with learning disabilities has been well established. This paper aims to explore the benefits of a therapy dog as part of a multi-disciplinary brain injury rehabilitation team. Methods: A qualitative survey of client and staff perspectives of the benefits of a therapy dog (Lambchop) was conducted based on the WHO-ICF framework. Clients participated in a holistic rehabilitation programme with a therapy dog as a key component of the compassionate mind approach to brain injury rehabilitation that posits activation of the soothing systems as a key element of rehabilitation including mood management. Results: Clients' perceived benefits of a therapy dog were mapped on to the WHO-ICF framework. At the impairment level the therapy dog supported cognitive abilities including attention, visuospatial abilities, route finding, memory and executive functioning. Activities sub-serving this included dog walking and dog discipline. Social participation level benefits included outings and walking the dog. The therapy dog served 
as a model for therapeutic art activities and was a key feature on children's Understanding Brain Injury day. Conclusions: Most clients and their families enjoyed having a therapy dog in the centre, supporting the value of therapy animals in rehabilitation.

Correspondence: Jill Winegardner; Jill.winegardner@ozc.nhs.uk

\title{
Behavioral problems after acquired brain injury: effects of the ABC-method
}

Winkens, leke'; Van Heugten, Caroline ${ }^{1,2}$; Ponds, Rudolf ${ }^{1,3}$ and Schrijnemaekers, AnneClaire $^{4}$

${ }^{1}$ Department of Psychiatry and Neuropsychology; Maastricht University; Maastricht; The Netherlands

${ }^{2}$ Department of Neuropsychology and Psychopharmacology; Maastricht University; Maastricht; The Netherlands

${ }^{3}$ Department of Psychiatry and Psychology, Maastricht University Medical Center (MUMC)/Alzheimer Center Limburg; Maastricht; The Netherlands

${ }^{4}$ Adelante; Hoensbroek; The Netherlands

$\boldsymbol{R}$ ackground and aims: For patients with severe behavioral problems after $\mathcal{B}$ acquired brain injury, treatment within regular rehabilitation settings often is not possible. The disturbing behavior causes burden for professionals and prevents patients from participating in treatment modules. The AntecedentBehavior-Consequence method (ABC-method) helps professionals to understand the origin of problem behavior and the impact of their own behavior on patients. This study sought to determine whether implementation of the ABC-method leads to a reduction in behavioral problems and burden experienced by professionals. Methods: Professionals of the mental healthcare institution Huize Padua (Boekel, the Netherlands) were trained in working with the ABC-method. Group treatment effects $(n=12)$ were examined on the Neuropsychiatric Inventory (NPI) and the Staff Observation Aggression Scale-Revised (SOAS-R). Individual treatment effects were studied using three Single Case Experimental AB-Designs. Results: A series of $t$-tests for paired samples showed no statistically significant short-term group effects in terms of reduction in problem behaviors on the SOAS-R $(t=.27 ; p=.79)$ and the NPI $(t=-.66 ; p=.53)$, or in level of burden experienced by professionals on the NPI $(t=1.22 ; p=.25)$. However, the mean SOAS-R score declined from 14.08 (pre-treatment) to 3.75 (post-treatment). The mean burden-score on the NPI declined from 5.92 (pre-treatment) to 3.75 (post-treatment). Conclusions: Preliminary data analysis shows no statistically significant effects of the ABC-method, although the trend in results seems promising. Follow-up measures are currently being conducted, and the research is being extended to other mental healthcare institutions in the Netherlands. Follow-up results and results from single case experimental designs will be presented.

Correspondence: Ieke Winkens; i.winkens@maastrichtuniversity.nl

\section{A new interdisciplinary postgraduate training programme in brain injury rehabili- tation: concept, delivery and evaluation}

\author{
Worthington, Andrew ${ }^{1}$ \\ ${ }^{7}$ Headwise \& Department of Psychology, University of Birmingham, UK
}

$\boldsymbol{R}$ ackground and aims: In the UK and internationally there is a recognised need for a better trained and more appropriately qualified workforce 
in the field of brain injury rehabilitation. Existing intra-professional training is not well suited to the complex nature of disability after brain injury and the inter-disciplinary nature of rehabilitation practice. There are no nationally recognised standards for training in this burgeoning field. This part-time course was developed to provide a unique opportunity for professionals from diverse backgrounds to learn together in a trans-disciplinary training course which combines the latest scientific understanding of brain function and recovery with practical application, while addressing the increasingly complex legal, social and ethical climate in which practitioners work. Method: The course is delivered by clinicians, lawyers and people with a brain injury. Students are taught using the same methods used in rehabilitation practice such as repetition, procedural learning, errorless learning, and spaced retrieval. Evaluation is by case reports, oral presentations and critical essays. Results: Challenges in setting up the course are discussed. Initial feedback has been extremely positive from students, contributors and external reviewers. The course is now attracting interest from the EU and beyond which will bring new challenges to ensure it maintains its standards for excellence. Conclusion: The popularity of the course has confirmed that there is a clear need for interdisciplinary training in brain injury rehabilitation which is not provided by individual professional organisations. This course provides one template for such a programme.

Correspondence: Andrew Worthington; aworthington@headwise.org.uk

\title{
Increased self-control, reduced aggression and adjustment in self-awareness to improve psychosocial integration: A single case study
}

\author{
García Sánchez, Olga ${ }^{1}$ and González Agudo, Cristina ${ }^{1}$ \\ ${ }^{1}$ Center of Rehabilitation of acquired brain injury, Polibea, C/ Ronda de Sobradiel, 36 28043. Madrid, Spain
}

\begin{abstract}
$B$ ackground and aims: Poor impulse control is a common feature in patients with traumatic brain injury (TBI) and often results in poor judgment, increased irritability, verbal and physical aggression. Such behaviours can have a negative impact on rehabilitation processes and the social outcomes resulting from TBI. The objective of this study was to describe a behavioural intervention to reduce aggression and increase self-control after TBI. Method: A case report is presented of a 59 year old man attending a rehabilitation unit over 3 years. Neuropsychological assessment included tests of memory and executive function, Neurobehavioral Rating Scale - Revised, Frontal System Behaviour Rating Scale and Self Awareness of Deficits Interview. The frequency of aggressive episodes and self- and family ratings were collected every 3 months throughout treatment and at 6 months follow-up. His self-control disorder was treated using behavioural strategies such as using keywords to reduce aggression and generating routines to increase activity engagement. Results: Analysis of data indicated a decline in the client's frequency of aggressive episodes and increased participation in his daily routine after the intervention commenced. The results also suggested that the intervention had a positive effect on how the client interacted with other people. Discussion and conclusions: These findings support that behavioural programs have promising utility for treating psychosocial dysfunction TBI.
\end{abstract}

Correspondence: Olga Garcia Sánchez; olgagarsan@hotmail.com. 